\title{
A PARTIAL INTEGRODIFFERENTIAL EQUATION IN GRANULAR MATTER AND ITS CONNECTION WITH A STOCHASTIC MODEL*
}

\author{
NOUREDDINE IGBIDA ${ }^{\dagger}$
}

\begin{abstract}
Our aim is to introduce and study a new partial integrodifferential equation (PIDE) associated with the dynamics of some physical granular structure with arbitrary component sizes, like a sandpile or sea dyke. Our PIDE is closely related to the nonlocal evolution problem introduced in [F. Andreu et al., Calc. Var. Partial Differential Equations, 35 (2009), pp. 279-316] by studying the limit, as $p \rightarrow \infty$, of the nonlocal $p$-Laplacian equation. We also show the connection between our PIDE and the stochastic model introduced by Evans and Rezakhanlou in [L. C. Evans and F. Rezakhanlou, Comm. Math. Phys., 197 (1998), pp. 325-345.] for modeling the sandpile problem.
\end{abstract}

Key words. partial integrodifferential equation (PIDE), physical granular structure, granular matter, nonlocal evolution problem, nonlocal $p$-Laplacian, growing sandpile, particle system, stochastic model for sandpile

AMS subject classifications. 35B40, 45A07, 45G10, 60J28

DOI. $10.1137 / 100810678$

1. Introduction. Following Aranson and Tsimring [6], we can define a granular material as a collection of discrete macroscopic solid grains of large size so that Brownian motion is irrelevant. Activated by external forces (gravity, electric and magnetic fields) a granular system may exhibit a transition from a granular solid to a liquid generating particulate flows which are central to a large number of modern applications. The description of such flows still represents a major challenge for the theory. In the last decade, several studies have been devoted to the mathematical and numerical studies of granular systems subjected to the gravity forces. Different models have been proposed using a kinetic approach $[9,10]$, cellular automata $[19,35,23,30]$, or partial differential equations $[7,34,15,28,8,20,22,21,24,18,29]$.

Most of the popular approaches that have recently attracted a renewed interest from the PDE community are based on partially fluidized granular flows (cf. [6]). In such flows some grains pass each other while other grains maintain static contacts with their neighbors. A typical example of granular system is the growing sandpile: the sand is poured on a flat surface according to a nonnegative function $f(t, x)$ (the source) and the pile grows in height. Using essentially the repose angle $\alpha$, which is the steepest angle that the surface of the structure makes with the ground (depending on the physical properties of the granular matter), nonlinear PDEs have been used to describe the dynamics of the growing sandpile. For instance, Bouchaud, Cates, Ravi Prakash, and Edwards introduced the so-called BCRE model (cf. [15] and [28]), a two-phase description of granular flow, one phase corresponding to rolling grains and the other phase to static ones. This model relies on PDE coupling phenomenological equations of transport and eikonal type. Using again mainly the repose angle, Prigozhin in [34] and independently Aronson, Evans, and Wu in [7] introduced a critical state model to

*Received by the editors October 5, 2010; accepted for publication (in revised form) February 24, 2012; published electronically June 5, 2012. This work was supported by the Spanish MEC and FEDER, project MTM2008-03176, and by "FLUPARTI" project (supported by "Conseil Régional de Picardie").

http://www.siam.org/journals/sima/44-3/81067.html

${ }^{\dagger}$ Institut de recherche XLIM-DMI, UMR-CNRS 7252, Faculté des Sciences et Techniques, Université de Limoges 123, Avenue Albert Thomas, 87060 Limoges, France (noureddine.igbida@unilim.fr). 
describe the dynamics of the growing sandpile, that is, the so-called evolution surfaces equation. It corresponds to a nonlinear evolution equation of $p$-Laplacian type with $p=\infty$. For completeness let us give a brief description of this model. We denote by $u=u(t, x)$ the height of the pile at time $t$ and at the position $x \in \mathbb{R}^{2}$. The function $u(t)$ grows with a slope which is always lower than the characteristic value $\tan (\alpha)$, i.e.,

$$
|\nabla u| \leq \tan (\alpha) .
$$

Moreover, using the continuity equation, the steepest descent property of the flow, and the fact that the dynamics vanishes when the angle of the surface is less (strictly) than $\alpha$, we obtain the following dynamics equation for $u$ (evolution surfaces equation):

$$
\left\{\begin{array}{l}
\partial_{t} u+\nabla \cdot \Phi=f, \Phi=-m \nabla u \\
m \geq 0, m(|\nabla u|-\tan (\alpha))=0
\end{array}\right.
$$

However, these approaches (BCRE model and evolution surface equation) seem to be reserved to the case of very small components (fine grains). Indeed, the solutions are regular and cannot correspond to general situations of nonsmall components for which the profile may be discontinuous. For arbitrary components, the dynamics is generated by the fact that the components (grains, blocks, etc.) move both by rolling down the slope or jumping from position to neighboring position if they are not supported by others. So, the state at any position is changing according to the states of its neighbors including the position itself.

A pioneering approach for describing such dynamics goes back to Evans and Rezakhanlou in 1998. The authors use particle systems and give a stochastic description of the dynamics (cf. [23]). In this situation, even if the source is deterministic, the trajectories are randomly selected by the blocks, so that the profile is a Markov process. The connection with the evolution surfaces equation appears when the blocks tend to be very small and very numerous (see [23] and [30]). Furthermore, recently in [3] (see also [4]) the authors introduced a new deterministic approach using nonlocal interactions to describe the dynamics of granular systems. It corresponds to a nonlocal evolution equation of $p$-Laplacian type with $p=\infty$. Moreover, by rescaling the problem in an appropriate way, they show that the solution of their model converges to the solution of the evolution surfaces equation. Our aim here is to consider the nonlocal interactions in an appropriate way to construct a new partial integrodifferential equation (PIDE) to model the dynamics of granular structures. We prove that our PIDE is well posed (existence and uniqueness of a solution) and show its connection with the stochastic model of Evans and Rezakhanlou as well as the nonlocal model of [3].

PIDEs have been used to model very different applied situations, for example, in biology [17, 25, 33], image processing [27, 32], particle systems [14], coagulation models [26], nonlocal anisotropic models for phase transition [1,2], and mathematical finance using optimal control theory [13]. One of their main interests is the connections with the stochastic process. For instance, in some situations, they give the distribution of the density of a random variable (assuming the process has a density) for stochastic process with discontinuous trajectories. For the connection between nonlocal partial differential equations and probability, see [5].

The paper is organized in three sections. The next section deals with the main results and is organized into two parts. In the first part, we establish the PIDE to model the dynamics of a granular structure and state the results of existence and 
uniqueness of the solution. In the second part, we recall the stochastic model of Evans and Rezakhanlou and give its connection to our PIDE. Section 3 is devoted to the study of the stationary equation associated with our PIDE. Finally, in the last section we give the proofs of the main results.

\section{Main results.}

2.1. The PIDE for granular matter. To introduce our model, we consider a granular matter with arbitrary components (grain, blocks, etc.). We pour the matter on a flat surface according to a nonnegative function $f(t, x)$ (the source), and we focus on the growing of the pile height denoted by $u=u(t, x)$ (at time $t$ and on the position $x \in \mathbb{R}^{2}$ ). First, we claim that the evolution of $u$ is given by the integral equation

$$
\frac{\partial u(t, x)}{\partial t}+\int_{\mathbb{R}^{2}} \mathcal{F}(t, x, y) d y=f(t, x) \quad \text { for any }(t, x) \in(0, T) \times \boldsymbol{R}^{2},
$$

where $\mathcal{F}(t, x, y)$ is an antisymmetric function, defined on $(0, T) \times \boldsymbol{I R}^{2} \times \boldsymbol{R}^{2}$, covering the exchanges between position $x$ and the nearby position $y$. Indeed, for a fixed position $x \in \boldsymbol{R}^{2}$ and a small time $\Delta t$, the evolution of $u$ is given by

$$
u(t+\Delta t, x) \simeq u(t, x)+\Delta t Q(t, x),
$$

where $Q(t, x)$ is the rate of materials arriving at the position $x$. Having in mind the external source $f, Q$ is given by

$$
Q(t, x)=f(t, x)+\operatorname{In}(t, x)-O u t(t, x),
$$

where $\operatorname{In}(t, x)$ records the blocks arriving at position $x$ from neighboring positions and Out $(t, x)$ records the blocks moving from position $x$ towards neighboring positions. Denoting by $j(t, x, y)$ the amount of cubes that moves from position $x$ to position $y$, we have

$$
\operatorname{In}(t, x)=\int_{\mathbb{R}^{2}} j(t, y, x) d y \quad \text { and } \quad \text { Out }(t, x)=\int_{\mathbb{R}^{2}} j(t, x, y) d y .
$$

So, setting

$$
\mathcal{F}(t, x, y)=j(t, x, y)-j(t, y, x),
$$

we have $\mathcal{F}(t, x, y)=-\mathcal{F}(t, y, x)$ and

$$
u(t+\Delta t, x) \simeq u(t, x)+\Delta t f(t, x)-\Delta t \int_{\mathbb{R}^{2}} \mathcal{F}(t, x, y) d y .
$$

At last, letting $\Delta t \rightarrow 0$, we obtain (2.1).

To close the problem, we need to give the connection between $\mathcal{F}$ and $u$. Our model is again a critical state model. However, in contrast with the case of small components where the angle of stability is the crucial factor, in our situation we use the nonlocal constraint of stability,

$$
|u(x)-u(y)| \leq \delta \quad \text { for } \quad|x-y| \leq \varepsilon,
$$

where $\delta>0$ and $\varepsilon>0$ are given constants depending on the gravity, the contact between the blocks, and their geometry. Condition (2.3) measures the size of irregularities of the cells. Recall that a similar constraint was already used in [3] with $\delta=\varepsilon$. 
Indeed, this natural constraint was obtained by letting $p$ go to infinity in the nonlocal $p$-Laplacian equation. Having in mind (2.3) and since the blocks of the structure move only when the limiting condition is turning to be violated, the dynamics in turn is concentrated on the set

$$
\left\{(t, x, y) \in(0, T) \times \boldsymbol{I R}^{2} \times \boldsymbol{R}^{2} ;|u(t, x)-u(t, y)|=\delta \text { and }|x-y| \leq \varepsilon\right\} .
$$

So, for any $t \in(0, T)$, we have

$$
\operatorname{Support}(\mathcal{F}(t)) \subseteq\left\{(x, y) \in \mathbb{R}^{2} \times \boldsymbol{R}^{2} ;|u(t, x)-u(t, y)|=\delta \text { and }|x-y| \leq \varepsilon\right\} \text {. }
$$

Moreover, since the blocks move by falling from high positions to lower positions, then the quantities $\mathcal{F}(t, x, y)$ and $u(x)-u(y)$ have the same sign. So, combining (2.4) and (2.2), we obtain

$$
\mathcal{F}(t, x, y)=|\mathcal{F}(t, x, y)| \frac{u(x)-u(y)}{\delta}
$$

Setting

$$
\mu(t, x, y)=\frac{1}{\delta}|\mathcal{F}(t, x, y)|
$$

leads immediately to the fact that $u$ satisfies the following PIDE:

$$
\left\{\begin{array}{l}
\frac{\partial u(t, x)}{\partial t}+\int_{\mathbb{R}^{2}}(u(t, x)-u(t, y)) \mu(t, x, y) d y=f(t, x) \quad \text { for any }(t, x) \in Q, \\
|u(t, x)-u(t, y)| \leq \delta \text { for any }|x-y| \leq \varepsilon \text { and } t \in(0, T), \\
\mu(t) \geq 0, \mu(t) \text { is symmetric for any } t \in(0, T), \\
\operatorname{Support}(\mu(t)) \subseteq\left\{(x, y) \in \mathbb{R}^{2} \times \mathbb{R}^{2} ;|u(t, x)-u(t, y)|=\delta \text { and }|x-y| \leq \varepsilon\right\},
\end{array}\right.
$$

where $Q:=(0, T) \times \boldsymbol{I R}^{2}$. Here, it is clear that the evolution of $u$ at a point $x$ and at time $t$ does not depend only on $u(t, x)$, but also on all the values of $u$ on its neighborhood, making the equation nonlocal. Recall that in standard PIDE the kernel $\mu$ is known and is "smooth." Here, our PIDE is nonstandard, since the computation of $u$ is related to the unknown kernel $\mu$ which is a Radon measure in general (see Theorem 2.1). So the first (resp., second) term of the integral operator reads $\int_{\mathbb{R}^{2}} u(t, y) \mu(x, d y)$ (resp., $\left.\int_{\mathbb{R}^{2}} u(t, x) \mu(t, x, d y)\right)$ and records the amount of material arriving at the position $u(t, x)$ from all other places (resp., leaving location $u(t, x)$ to travel to all other sites).

To simplify the notation, let us introduce the set of admissible profiles,

$$
K_{\varepsilon}^{\delta}=\left\{z \in L^{2}\left(\boldsymbol{R}^{2}\right) ;|z(x)-z(y)| \leq \delta \text { for }|x-y| \leq \varepsilon\right\},
$$

and, for any $z \in K_{\varepsilon}^{\delta}$, we denote

$$
\mathcal{R}_{\varepsilon}^{\delta}(z)=\left\{(x, y) \in \boldsymbol{R}^{2} \times \boldsymbol{R}^{2} ;|z(x)-z(y)|=\delta \text { and }|x-y| \leq \varepsilon\right\} .
$$

Taking $u_{0}$ as an initial value at $t=0$, our PIDE reads

$$
\left(P_{\varepsilon}^{\delta}\right) \begin{cases}\frac{\partial u(t, x)}{\partial t}+\int_{\mathbb{R}^{2}}(u(t, x)-u(t, y)) \mu(t, x, d y)=f(t, x) & \text { for }(t, x) \in Q, \\ u(t) \in K_{\varepsilon}^{\delta}, \quad \mu(t) \geq 0, \quad \mu(t)\left\llcorner\mathcal{R}_{\varepsilon}^{\delta}(u(t))\right. & \text { for } t \in[0, T), \\ u(0)=u_{0}, & \end{cases}
$$


where we assume that, for any $t \in(0, T), \mu(t)$ is a Radon measure, $u(t) \in \mathcal{C}_{0}\left(\boldsymbol{I R}^{2}\right)$, and we use the notation $\mu(t)\left\llcorner\mathcal{R}_{\varepsilon}^{\delta}(u(t))\right.$ to denote that $\operatorname{Support}(\mu(t)) \subseteq \mathcal{R}_{\varepsilon}^{\delta}(u(t))$.

To set our results of existence and uniqueness of a solution for $\left(P_{\varepsilon}^{\delta}\right)$, we consider

$$
\begin{aligned}
\mathcal{M}_{b}^{s}\left(\boldsymbol{R}^{2} \times \boldsymbol{R}^{2}\right)^{+} & :=\left\{\mu \in \mathcal{M}_{b}\left(\boldsymbol{R}^{2} \times \boldsymbol{R}^{2}\right)^{+} ; \iint \xi(x, y) \mu(d x, d y)\right. \\
& \left.=\iint \xi(y, x) \mu(d x, d y) \text { for any } \xi \in \mathcal{C}_{c}\left(\boldsymbol{R}^{2} \times \boldsymbol{I R}^{2}\right)\right\},
\end{aligned}
$$

where we use $\mu(d x, d y)$ to denote $d \mu(x, y)$.

TheOREM 2.1. Assume that $f \in B V\left(0, T ; L^{2}\left(\boldsymbol{I R}^{2}\right)\right) \cap L^{1}\left(0, T ; \mathcal{C}_{0}\left(\boldsymbol{I R}^{2}\right)\right)$ and that there exists $\alpha \in L^{1}(0, T)$ such that, for any $h \in \mathbb{R}^{N}$, we have

$$
\sup _{x \in \mathbb{R}^{2}}|f(t, x+h)-f(t, x)| \leq \alpha(t), \quad \text { a.e. } t \in(0, T) .
$$

Then, for any $u_{0} \in K_{\varepsilon}^{\delta} \cap \mathcal{C}_{0}\left(\boldsymbol{R}^{2}\right),\left(P_{\varepsilon}^{\delta}\right)$ has a unique solution $u$ in the sense that $u \in W^{1, \infty}\left(0, T ; L^{2}(\Omega)\right) \cap L^{\infty}\left(0, T ; \mathcal{C}_{0}\left(\boldsymbol{R}^{2}\right)\right), u(0)=u_{0}$, for any $t \in(0, T), u(t) \in K_{\varepsilon}^{\delta}$ and there exists $\mu(t) \in \mathcal{M}_{b}^{s}\left(\boldsymbol{I R}^{2} \times \boldsymbol{R}^{2}\right)^{+}$such that

$$
\mu(t)\left\llcorner\mathcal{R}_{\varepsilon}^{\delta}(u(t))\right.
$$

and

$$
\int_{\mathbb{R}^{2}} \int_{\mathbb{R}^{2}} \xi(x)(u(t, x)-u(t, y)) \mu(t, d x, d y)=\int_{\mathbb{R}^{2}}\left(f(t, x)-\frac{\partial u(t, x)}{\partial t}\right) \xi(x) d x
$$

for a.e. $t \in(0, T)$ and for any $\xi \in \mathcal{C}_{0}\left(\boldsymbol{I R}^{2}\right) \cap L^{2}\left(\boldsymbol{I R}^{2}\right)$. Moreover, if for $i=1,2, u_{i}$ is the solution corresponding to $f_{i}$, then, for any $1 \leq q \leq \infty$, we have

$$
\frac{d}{d t}\left\|u_{1}-u_{2}\right\|_{L^{q}\left(\boldsymbol{R}^{2}\right)} \leq\left\|f_{1}-f_{2}\right\|_{L^{q}\left(\boldsymbol{R}^{2}\right)} \quad \text { in } \mathcal{D}^{\prime}(0, T)
$$

and

$$
\frac{d}{d t}\left\|\left(u_{1}-u_{2}\right)^{+}\right\|_{L^{1}\left(\boldsymbol{R}^{2}\right)} \leq\left\|\left(f_{1}-f_{2}\right)^{+}\right\|_{L^{1}\left(\boldsymbol{R}^{2}\right)} \quad \text { in } \mathcal{D}^{\prime}(0, T) .
$$

Now, let us denote by $\mathbb{I}_{K_{\varepsilon}^{\delta}}$ the convex indicator function of $K_{\varepsilon}^{\delta}$ defined by

$$
\mathbb{I}_{K_{\varepsilon}^{\delta}}(z)= \begin{cases}0 & \text { if } z \in K_{\varepsilon}^{\delta}, \\ +\infty & \text { otherwise. }\end{cases}
$$

The subdifferential of $\mathbb{I}_{K_{\varepsilon}^{\delta}}$ in $L^{2}(\Omega)$ is given by $v \in \partial \mathbb{I}_{K_{\varepsilon}^{\delta}}(g)$ if and only if $v \in K_{\varepsilon}^{\delta}$, $g \in L^{2}\left(\boldsymbol{R}^{2}\right)$, and

$$
\int_{\Omega} g(z-v) \leq 0 \quad \text { for any } z \in K_{\varepsilon}^{\delta} .
$$

Thanks to [16], we know that for any $u_{0} \in K_{\varepsilon}^{\delta}$ and $f \in B V\left(0, T ; L^{2}\left(\boldsymbol{I R}^{2}\right)\right)$, the evolution problem

$$
\left\{\begin{array}{l}
u_{t}+\partial \boldsymbol{I}_{K_{\varepsilon}^{\delta}}(u) \ni f \quad \text { in }(0, T), \\
u(0)=u_{0},
\end{array}\right.
$$


has a unique solution, in the sense that $u \in W^{1, \infty}\left(0, T ; L^{2}\left(\boldsymbol{R}^{2}\right)\right), u(0)=u_{0}$, and, for any $t \in(0, T), u(t) \in K_{\varepsilon}^{\delta}$ and

$$
\int_{\mathbb{R}^{2}}\left(f(t)-\frac{\partial u(t)}{\partial t}\right)(u(t)-z) \geq 0 \quad \text { for any } z \in K_{\varepsilon}^{\delta} .
$$

The connexion between the model $\left(P_{\varepsilon}^{\delta}\right)$ and the nonlinear dynamic (2.6) is given in the following theorem.

THEOREM 2.2. Under the assumptions of Theorem 2.1, $u$ is a solution of $\left(P_{\varepsilon}^{\delta}\right)$ if and only if $u$ is a solution of (2.6).

Remark 2.3.

1. Recall that if $\delta=\varepsilon \rightarrow 0$, it is proven in [3] that the solution $u_{\varepsilon}^{\delta}$ of (2.6) converges to the solution of the local sandpile problem

$$
\left\{\begin{array}{l}
\partial_{t} u+\partial \boldsymbol{I}_{K}(u) \ni f \quad \text { in }(0, T), \\
u(0)=u_{0}
\end{array}\right.
$$

where

$$
K=\left\{z \in W_{0}^{1,2}(\Omega) \cap W^{1, \infty}(\Omega) ;\|\nabla z\|_{L^{\infty}(\Omega)} \leq 1\right\} .
$$

2. In the case of Euclidean distance, it is known that the problem (2.7) is equivalent to the evolution surface model (1.1), where one needs to handle the problem in an appropriate way by taking into account the fact that $m$ is a measure (see, for instance, [31] and the references therein). So, by Theorem 2.2 and the previous remark, as $\varepsilon=\delta \rightarrow 0$, the solution $u_{\varepsilon}^{\delta}$ of $P_{\varepsilon}^{\delta}$ converges to $u$ the unique solution of the evolution surface model (1.1). As to the kernel $\mu_{\varepsilon}^{\varepsilon}$, it is not difficult to see that, by taking a subsequence $\varepsilon_{k} \rightarrow 0, \mu_{\varepsilon_{k}}$ converges in $\mathcal{M}_{b}\left(\boldsymbol{R}^{2}\right)$-weak ${ }^{*}$ to some nonnegative Radon measure $\mu$. However, the characterization of $\mu$ is an open problem; it should be the density $m$ but the proof is not clear yet for us.

3. One sees that $\left(P_{\varepsilon}^{\delta}\right)$ is a nonlocal coupled equation between $u$ and a kernel $\mu$. Thanks to Theorem 2.2, the uniqueness of the solution $u$ of $\left(P_{\varepsilon}^{\delta}\right)$ is connected to the uniqueness of the solution of an evolution problem governed by a subdifferential operator. As to the unknown Kernel $\mu$, we see that it has no equation but only a restriction on its support. We do not know if $\mu$ is unique or not, even if it is a regular function.

2.2. Evans-Rezakhanlou stochastic model for a sandpile. The aim of this section is to give the connection between the PIDE $\left(P_{\varepsilon}^{\delta}\right)$ and the Evans-Rezakhanlou stochastic model introduced in [23]. Let us recall briefly the model. The objective is to study the evolution of a stack of unit cubes resting on the plane when new cubes are being added to the pile, either placed upon an unoccupied square in the plane or upon the top of a current column.

Consider a set of sites labeled by a couple of integers $i=\left(i_{1}, i_{2}\right) \in \mathbb{Z}^{2}$. The source term is a deterministic function $\hat{f}:(0, T) \times \mathbb{Z}^{2} \rightarrow \boldsymbol{R}$ assigning cubes per unit of time on the sites $i \in \mathbb{Z}^{2}$. The evolution of the stack is specified by two rules for the added cubes:

- The cube is assigned to a position connected to several downhill "staircases" along which it can move, and the cube will randomly select among the available downhill paths. 


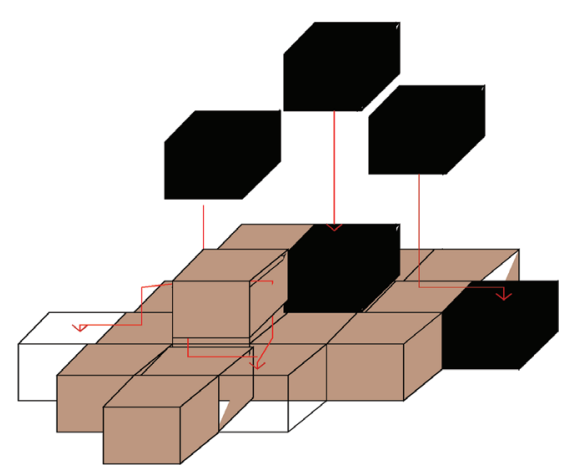

FIG. 2.1. Growing pile of cubes.

- The assigned cube has no "staircases" derived from the position it was put on and remains in place.

A staircase is a chain of adjacent positions with a height difference equal to one cube. Here, we assume that a cube moves by falling in one of the four directions (forward, back, left, or right) in order to get a stable configuration, which means that the heights of any two adjacent columns of cubes can differ by at most one.

Even if the source is deterministic, it is clear here that one needs to use a stochastic approach to describe the evolution of the height of the pile. We consider the lattice $\mathcal{O}:=\mathbb{Z}^{2}$ equipped with the norm

$$
|i|=\left|i_{1}\right|+\left|i_{2}\right|,
$$

and we write $i=\left(i_{1}, i_{2}\right) \in \mathbb{Z}^{2}$ to denote a typical site in $\mathbb{Z}^{2}$. We say $i, j \in \mathbb{Z}^{2}$ are adjacent, written $i \sim j$, provided

$$
|i-j| \leq 1
$$

Then we consider the Hilbert space

$$
H:=l^{2}\left(\mathbb{Z}^{2}\right)=\left\{\eta: \mathbb{Z}^{2} \rightarrow \boldsymbol{I R} ;\|\eta\|:=\sum_{i \in \mathbb{Z}^{2}} \eta(i)^{2}<\infty\right\} .
$$

A (stable) configuration is a mapping $\eta: \mathbb{Z}^{2} \rightarrow \mathbb{Z}$ such that

$$
\left\{\begin{array}{l}
|\eta(i)-\eta(j)| \leq 1 \quad \text { if } i \sim j \\
\text { and } \eta \text { has bounded support. }
\end{array}\right.
$$

The state space is the subspace of $H$ given by

$$
S:=\left\{\eta: \mathbb{Z}^{2} \rightarrow \mathbb{Z} ; \eta \text { is a configuration }\right\} .
$$

To simplify the presentation, we assume throughout this section that $\hat{f}$, the function controlling the rate new cubes are added to the pile (or removed from it if $f$ has negative part), is nonnegative.

By depositing the cubes with a rate $\hat{f}$, we generate a stochastic process $(\eta(t), t \geq$ 0 ) in the state space $S$. It is clear that the probability that $\eta(t)$ is situated (at time $t$ ) in a given set $\Gamma$ of $S$ under the condition that the movement of the system up 
to time $s(s<t)$ is completely known and depends only on the state of the system at time $s$. In other words $(\eta(t), t \geq 0)$ is a Markov process. To study the process $(\eta(t), t \geq 0)$, we need to know its infinitesimal generator $A$. To this aim, the authors of [23] consider $p(i, j, \xi)$ the probability that a cube placed on a given configuration $\xi \in S$ at the position $i$ will end up at $j$ after it has fallen downward over the stack $\xi$. So, for any $i, j \in \mathbb{Z}^{2}$ we have

$$
0 \leq p(i, j, \xi) \leq 1 \quad \text { and } \quad \sum_{j \in \mathbb{Z}^{2}} p(i, j, \xi)=1
$$

Furthermore, they introduce the factor $c(j, \eta, \tau)$ (a highly nonlocal factor) recording the rate at which, at time $\tau$, new cubes come to rest at the site $j$ after falling downhill. The parameter $c$ is given by

$$
c(j, \eta(t), t)=\sum_{i \in \mathbb{Z}^{2}} p(i, j, \eta(t)) \hat{f}(t, i) \quad \text { for any }(t, j) \in \mathbb{Z}^{2} \times[0, \infty)
$$

and satisfies

$$
\sum_{j \in \mathbb{Z}^{2}} c(j, \eta(t), t)=\sum_{i \in \mathbb{Z}^{2}} \hat{f}(t, i)
$$

Let us denote by $B(S)$ the set of bounded functions defined on $S$. Thanks to [23], the infinitesimal generator of the Markov process $(\eta(t), t \geq 0)$ is given by $\mathcal{L}_{t}: F \in$ $B(S) \rightarrow \mathcal{L}_{t} F \in B(S)$ the linear time dependent operator defined by

$$
\left(\mathcal{L}_{t} F\right)(\xi):=\sum_{j \in \mathbb{Z}^{N}} c(j, \xi, t)\left(F\left(T_{j}(\xi)\right)-F(\xi)\right)
$$

for any $t>0$ and $\xi \in S$, and

$$
T_{j}: \xi \in S \rightarrow T_{j}(\xi) \in S \quad \text { with } \quad T_{j}(\xi)(i)=\left\{\begin{array}{l}
\xi(i)+1 \text { if } i \sim j, \\
\xi(i) \text { otherwise } .
\end{array}\right.
$$

To give the connection between the PIDE $\left(P_{\varepsilon}^{\delta}\right)$ and the Markov process $(\eta(t), t \geq$ 0 ), we set $\delta=\frac{1}{P}, \varepsilon=\frac{1}{N}$ for $P, N \in \mathbb{I N}^{*}$, and we assume the following:

H1. $u_{0} \equiv 0$.

H2. $f \in B V\left(0, T ; L^{2}\left(\boldsymbol{R}^{2}\right)\right)$ is such that $f(t)$ is nonnegative and compactly supported for any $t \in[0, T)$.

H3. $\hat{f}:(0, T) \times \mathbb{Z}^{2} \rightarrow \boldsymbol{R}$ is given by

$$
\hat{f}(t, i)=f\left(\frac{t}{P}, \frac{i}{N}\right) \quad \text { for any }(t, i) \in[0, \infty) \times \mathbb{Z}^{2} .
$$

We introduce the stochastic process $\left(\eta_{P, N}(t), t \geq 0\right)$ given by

$$
\eta_{P, N}(t, x)=\frac{1}{P} \eta(P t,[N x]) \quad \text { for any } t \geq 0 \text { and } x \in \mathbb{R}^{2},
$$

where $(\eta(., t), t \geq 0)$ is the Markov process generated by $\hat{f}$. Notice that $\eta_{P, N}$ describes the random evolution of eight of the structure of blocks whose base is a square of side length $\varepsilon=1 / N$ and height length $\delta=1 / P$. 
THEOREM 2.4. Under the assumption $\mathrm{H} 1-\mathrm{H} 3$, let $u$ be the solution of (2.6), where $\varepsilon=1 / N, \delta=1 / P, N, P \in \boldsymbol{I N}^{*}$, and $\boldsymbol{R}^{2}$ is equipped with the norm

$$
\left|\left(x_{1}, x_{2}\right)\right|=\left|x_{1}\right|+\left|x_{2}\right| \quad \text { for any }\left(x_{1}, x_{2}\right) \in \mathbb{R}^{2} .
$$

For any $t \in(0, T)$, we have

$$
\begin{array}{r}
\left(\boldsymbol{E}\left[\int_{\boldsymbol{R}^{2}}\left|u(t, x)-\eta_{P, N}(t, x)\right|^{2}\right]\right)^{\frac{1}{2}} \leq \delta^{\frac{1}{2}}\left(\int_{0}^{t} \int_{\mathbb{R}^{2}} f\left(s, \frac{[N x]}{N}\right) d x d s\right)^{\frac{1}{2}} \\
+\left(\int_{0}^{t} \int_{\mathbb{R}^{2}}\left|f(t, x)-f\left(s, \frac{[N x]}{N}\right)\right|^{2} d x d s\right)^{\frac{1}{2}}
\end{array}
$$

where

$$
[x]=\left(\left[x_{1}\right],\left[x_{2}\right]\right) \quad \text { for any } x=\left(x_{1}, x_{2}\right) \in \boldsymbol{I R}^{2},
$$

and, for $i=1,2,\left[x_{i}\right]$ denotes the integer part of the real number $x_{i}$.

A typical situation for Theorem 2.4 corresponds to the case where $f(t)$ is constant on each set $I_{i}:=\left\{x \in \mathbb{R}^{2} ;[N x]=i\right\}$, with $i \in \mathbb{Z}^{2}$; i.e.,

$$
f(t, x)=f\left(t, \frac{[N x]}{N}\right) \quad \text { for any }(t, x) \in(0, \infty) \times \boldsymbol{I R}^{2} .
$$

In such situation, we imagine that the source is assigning, on the sites $I_{i}$, blocks whose base is a square of side length $\varepsilon=1 / N$ and height length $\delta=1 / P$. In particular, we have the following.

COROLLARY 2.5. Under the assumptions of Theorem 2.4, we assume moreover that (2.13) is fulfilled. Then, for any $t \in(0, T)$, we have

$$
\boldsymbol{I E}\left[\int_{\mathbb{R}^{2}}\left|u(t, x)-\eta_{P, N}(t, x)\right|^{2}\right] \leq \delta \int_{0}^{t} \int_{\mathbb{R}^{2}}|f(s, x)| d x d t .
$$

Remark 2.6.

1. In general, the norm of $\boldsymbol{R}^{2}$ is taken arbitrary in the formulation of $\left(P_{\varepsilon}^{\delta}\right)$. However, in Theorem $2.4, \boldsymbol{R}^{2}$ is equipped with the norm (2.12) according to the $l^{1}$-norm of $\mathbb{Z}^{2}$. This is related to the movements we assign for the cubes (forward, back, left, or right). Now, if we enable the cubes to move in the eight directions by adding the displacements on the diagonal, then the results of the theorem remain true by equipping $\mathbb{Z}^{2}$ with the norm $\left|\left(i_{1}, i_{2}\right)\right|_{\infty}=$ $\max \left(\left|i_{1}\right|,\left|i_{2}\right|\right)$ and $\boldsymbol{I R}^{2}$ with the corresponding norm $\left|\left(x_{1}, x_{2}\right)\right|_{\infty}=\max \left(\left|x_{1}\right|,\left|x_{2}\right|\right)$ for any $\left(x_{1}, x_{2}\right) \in \boldsymbol{I R}^{2}$.

2. Theorem 2.4 implies that the solution of the PIDE is a deterministic approximation of the random height $\eta(t)$ for any $t \in(0, T)$. Indeed, letting $(\varepsilon, \delta) \rightarrow(0,0)$, we see that $u$ and $\eta_{P, N}$ coincide. In particular, in the case where $\varepsilon=\delta \rightarrow 0$, thanks to [3] and [23], $u$ and $\eta_{P, N}$ coincide with the solution of the local sandpile problem (2.7).

3. For simplification, we take here the initial data equal to $\eta(0) \equiv 0$ and $u_{0} \equiv 0$. Similar results may be proven for general initial data by rescaling it and using the same techniques of this paper.

4. By using the fact that for $\eta: \mathbb{Z}^{2} \rightarrow \mathbb{Z}$ and $u(t,):. \mathbb{R}^{2} \rightarrow \boldsymbol{R}$ the solutions of (2.7) are Lipschitz continuous, it is proven in [23] that the convergence of $\eta_{P, N}$ to $u$ holds true in $\mathcal{C}\left(\boldsymbol{R}^{2}\right)$ as $\varepsilon=\delta=1 / N \rightarrow 0$. 
3. Stationary PIDE. To study $\left(P_{\varepsilon}^{\delta}\right)$, we use nonlinear semigroup theory associated with an evolution equation in a Hilbert space. This approach connects the study of the evolution equation with the stationary equation associated with the Euler implicit discretization in time. For any $\sigma>0$, we say that $\left(t_{i}, f_{i}\right)_{i=1, \ldots, n}$ is a $\sigma$-discretization of $\left(P_{\varepsilon}^{\delta}\right)$ if $t_{0}=0<t_{1}<\cdots<t_{n-1}<T=t_{n}$ with $t_{i}-t_{i-1}=\sigma$, $f_{1}, \ldots, f_{n} \in \mathcal{C}_{0}\left(\boldsymbol{I R}^{2}\right)$, such that

$$
\sum_{i=1}^{n} \int_{t_{i-1}}^{t_{i}}\left\|f(t)-f_{i}\right\|_{L^{2}\left(\boldsymbol{R}^{2}\right)} \leq \sigma .
$$

Moreover, we say that $u_{\sigma}$ is a $\sigma$-approximate solution of $\left(P_{\varepsilon}^{\delta}\right)$ if there exists $\left(t_{i}, f_{i}\right)_{i=1, \ldots, n}$ a $\sigma$-discretization such that

$$
u_{\sigma}(t)= \begin{cases}u_{0} & \text { for } \left.t \in] 0, t_{1}\right] \\ u_{i} & \text { for } \left.t \in] t_{i-1}, t_{i}\right], i=1, \ldots, n\end{cases}
$$

and $u_{i}$ solves the Euler implicit time discretization

$$
\left\{\begin{array}{l}
u_{i}(x)+\sigma \int_{\boldsymbol{R}^{2}}\left(u_{i}(x)-u_{i}(y)\right) \mu_{i}(x, d y)=\sigma f_{i}(x)+u_{i-1} \quad \text { for } x \in \boldsymbol{R}^{2} \\
u_{i} \in K_{\varepsilon}^{\delta}, \mu_{i} \in \mathcal{M}_{b}^{s}\left(\boldsymbol{R}^{2} \times \boldsymbol{R}^{2}\right)^{+}, \mu_{i} \mathbf{L} \mathcal{R}_{\varepsilon}^{\delta}\left(u_{i}\right)
\end{array}\right.
$$

for any $i=1, \ldots, n$. We see that problem (3.2) is a particular case of the generic stationary problem

$$
\left\{\begin{array}{l}
u(x)+\int_{\mathbb{R}^{2}}(u(x)-u(y)) \mu(x, d y)=f(x) \quad \text { for } x \in \mathbb{R}^{2}, \\
u \in K_{\varepsilon}^{\delta}, \mu \in \mathcal{M}_{b}^{s}\left(\boldsymbol{R}^{2} \times \boldsymbol{R}^{2}\right)^{+}, \mu\left\llcorner\mathcal{R}_{\varepsilon}^{\delta}(u),\right.
\end{array}\right.
$$

where we assume without lost of generality that $\sigma=1$. Here, $f$ is a given function in $L^{2}\left(\boldsymbol{I R}^{2}\right)$ and the couple $(u, \mu)$ is unknown. To prove existence and uniqueness of a solution for $\left(P_{\varepsilon}^{\delta}\right)$, we begin by studying the stationary problem (3.3).

Theorem 3.1. For any $f \in \mathcal{C}_{0}\left(\boldsymbol{R}^{2}\right) \cap L^{2}\left(\boldsymbol{I R}^{2}\right)$, (3.3) has a unique solution $u$ in the following sense:

$$
\left\{\begin{array}{l}
u \in K_{\varepsilon}^{\delta} \cap \mathcal{C}_{0}\left(\boldsymbol{R}^{2}\right), \exists \mu \in \mathcal{M}_{b}^{s}\left(\boldsymbol{I R}^{2} \times \boldsymbol{R}^{2}\right)^{+}, \mu\left\llcorner\mathcal{R}_{\varepsilon}^{\delta}(u),\right. \text { and } \\
\int_{\boldsymbol{R}^{2}} u(x) \xi(x) d x+\int_{\boldsymbol{R}^{2}} \int_{\boldsymbol{R}^{2}}(u(x)-u(y)) \xi(x) \mu(d x, d y) \\
=\int_{\boldsymbol{R}^{2}} f(x) \xi(x) d x
\end{array}\right.
$$

for any $\xi \in \mathcal{C}_{0}\left(\boldsymbol{R}^{2}\right) \cap L^{2}\left(\boldsymbol{R}^{2}\right)$. Moreover, $u$ is a solution of (3.4) if and only if

$$
u=\boldsymbol{P}_{K_{\varepsilon}^{\delta}}(f),
$$

where $\boldsymbol{P}_{K_{\varepsilon}^{\delta}}$ is the projection with respect to the $L^{2}\left(\boldsymbol{R}^{2}\right)$-norm on the convex $K_{\varepsilon}^{\delta}$.

The proof of Theorem 3.1 follows as a consequence of the following lemmas.

Lemma 3.2. Assume $f \in L^{2}\left(\boldsymbol{R}^{2}\right)$. If $u$ is a solution of $(3.3)$, then $u=\boldsymbol{P}_{K_{\varepsilon}^{\delta}}(f)$.

Proof. Let $u$ be a solution of (3.4). Let us prove that

$$
\int_{\mathbb{R}^{2}}(f(x)-u(x))(u(x)-z(x)) d x \geq 0 \quad \text { for any } z \in K_{\varepsilon}^{\delta} .
$$


For any $\lambda>0$, let us denote by $\rho_{\lambda}$ the standard mollifiers in $\boldsymbol{R}^{2}$, and by $z_{\lambda}=z * \rho_{\lambda}$ the usual regularizing of $z$ by convolution. Using $u-z_{\lambda}$ as a test function in the integral equation satisfied by $u$ in (3.4), we get

$$
\begin{aligned}
\int_{\mathbb{R}^{2}}(f(x)-u(x))(u(x)-z(x)) d x & =\lim _{\lambda \rightarrow 0} \int_{\mathbb{R}^{2}}(f(x)-u(x))\left(u(x)-z_{\lambda}(x)\right) d x \\
& =\lim _{\lambda \rightarrow 0} \int_{\mathbb{R}^{2}} \int_{\mathbb{R}^{2}}(u(x)-u(y))\left(u(x)-z_{\lambda}(x)\right) \mu(d x, d y) \\
& =: \lim _{\lambda \rightarrow 0} I_{\lambda} .
\end{aligned}
$$

It is not difficult to see that $z_{\lambda} \in K_{\varepsilon}^{\delta}$, so that, by using the fact that $\mu$ is symmetric, we have

$$
\begin{aligned}
I_{\lambda}= & \int_{\mathbb{R}^{2}} \int_{\mathbb{R}^{2}}(u(x)-u(y))^{2} \mu(d x, d y)+\int_{\mathbb{R}^{2}} \int_{\mathbb{R}^{2}}(u(x)-u(y))\left(u(y)-z_{\lambda}(y)\right) \mu(d x, d y) \\
& +\int_{\mathbb{R}^{2}} \int_{\mathbb{R}^{2}}(u(x)-u(y))\left(z_{\lambda}(y)-z_{\lambda}(x)\right) \mu(d x, d y) \\
= & \int_{\mathbb{R}^{2}} \int_{\mathbb{R}^{2}}(u(x)-u(y))^{2} \mu(d x, d y)-I_{\lambda} \\
& +\int_{\mathbb{R}^{2}} \int_{\mathbb{R}^{2}}(u(x)-u(y))\left(z_{\lambda}(y)-z_{\lambda}(x)\right) \mu(d x, d y) .
\end{aligned}
$$

This implies that

$$
\begin{aligned}
2 I_{\lambda}= & \int_{\mathbb{R}^{2}} \int_{\mathbb{R}^{2}}(u(x)-u(y))^{2} \mu(d x, d y) \\
& +\int_{\boldsymbol{R}^{2}} \int_{\mathbb{R}^{2}}(u(x)-u(y))\left(z_{\lambda}(y)-z_{\lambda}(x)\right) \mu(d x, d y) \\
\geq & \int_{\mathbb{R}^{2}} \int_{\mathbb{R}^{2}}(u(x)-u(y))^{2}\left(1-\frac{\left|z_{\lambda}(y)-z_{\lambda}(x)\right|}{|u(x)-u(y)|}\right) \mu(d x, d y) .
\end{aligned}
$$

Using the fact that $\mu$ is supported on the set $\left\{(x, y) \in \mathbb{R}^{2} \times \boldsymbol{R}^{2} ;|u(x)-v(y)|=\right.$ $\delta$ for $|x-y| \leq \varepsilon\}$, we obtain

$$
2 I_{\lambda} \geq \int_{\mathbb{R}^{2}} \int_{\mathbb{R}^{2}}(u(x)-u(y))^{2}\left(1-\frac{\left|z_{\lambda}(y)-z_{\lambda}(x)\right|}{\delta}\right) \mu(d x, d y) .
$$

At last, since $z_{\lambda} \in K_{\varepsilon}^{\delta}$ and $\operatorname{Support}(\mu(t)) \subset\left\{(x, y) \in \boldsymbol{I R}^{2} \times \boldsymbol{R}^{2} ;|x-y| \leq \varepsilon\right\}$, we deduce that $2 I_{\lambda} \geq 0$ and the proof is finished.

To prove the existence of a solution for (3.4), we use the nonlocal $p$-Laplacian equation

$$
u_{p}(x)+\int_{\mathbb{R}^{2}} J(x-y)\left|\frac{u_{p}(x)-u_{p}(y)}{\delta}\right|^{p-2}\left(u_{p}(x)-u_{p}(y)\right) d y=f(x) \text { for } x \in \mathbb{R}^{2}
$$


where $J \in \mathcal{C}\left(\boldsymbol{R}^{2}\right)$ is a nonnegative continuous radial function in $\boldsymbol{R}^{2}$ such that $\operatorname{Support}(J)=B(0, \varepsilon), J(0)>0$, and $\int_{\mathbb{R}^{2}} J(x) d x=1$. Thanks to [3], we know that for any $f \in L^{2}\left(\boldsymbol{R}^{2}\right) \cap L^{\infty}\left(\boldsymbol{R}^{2}\right)$, (3.7) has a unique solution $u_{p}$. Moreover, for any $1 \leq q \leq \infty$, we have

$$
\left\|u_{p}\right\|_{L^{q}\left(\boldsymbol{R}^{2}\right)} \leq\|f\|_{L^{q}\left(\boldsymbol{R}^{2}\right)}
$$

and, if $u_{i}$ is the solution corresponding to $f_{i} \in L^{2}\left(\boldsymbol{R}^{2}\right) \cap L^{\infty}\left(\boldsymbol{R}^{2}\right)$ for $i=1,2$, then

$$
\left\|u_{1}-u_{2}\right\|_{L^{q}\left(\boldsymbol{R}^{2}\right)} \leq\left\|f_{1}-f_{2}\right\|_{L^{q}\left(\boldsymbol{R}^{2}\right)}
$$

and

$$
\left\|\left(u_{1}-u_{2}\right)^{+}\right\|_{L^{1}\left(\boldsymbol{R}^{2}\right)} \leq\left\|\left(f_{1}-f_{2}\right)^{+}\right\|_{L^{1}\left(\boldsymbol{R}^{2}\right)} .
$$

Our aim is to prove that by passing to the limit in (3.7), we obtain the solution of (3.4).

First, let us give some estimates that will be useful in what follows.

Lemma 3.3. Assume $f \in L^{2}\left(\boldsymbol{I R}^{2}\right) \cap L^{\infty}\left(\boldsymbol{I R}^{2}\right)$ and let $u_{p}$ be the solution of (3.7). Then

$$
\frac{1}{\delta^{p-2}} \int_{\boldsymbol{R}^{2}} \int_{\boldsymbol{R}^{2}} J(x-y)\left|u_{p}(x)-u_{p}(y)\right|^{p} d x d y=2 \int_{\boldsymbol{R}^{2}}\left(f(x)-u_{p}(x)\right) u_{p}(x) d x
$$

and

$$
\frac{1}{\delta^{p-2}} \int_{\mathbb{R}^{2}} \int_{\mathbb{R}^{2}} J(x-y)\left|u_{p}(x)-u_{p}(y)\right|^{p} d x d y \leq 2 \int_{\mathbb{R}^{2}}|f(x)|^{2} d x .
$$

Moreover, for any $1 \leq q \leq \infty$, we have

$$
\left\|u_{p}(.+h)-u_{p}(.)\right\|_{L^{q}\left(\boldsymbol{R}^{2}\right)} \leq\|f(.+h)-f(.)\|_{L^{q}\left(\boldsymbol{R}^{2}\right)} \quad \text { for any } h \in \mathbb{R}^{2}
$$

and, for any $1 \leq q \leq p<\infty$, we have

$$
\begin{aligned}
& \int_{\mathbb{R}^{2}} \int_{\mathbb{R}^{2}} J(x-y)\left|u_{p}(x)-u_{p}(y)\right|^{q} d x d y \\
& \quad \leq\left(\int_{\mathbb{R}^{2}} \int_{\mathbb{R}^{2}} J(x-y)\left|u_{p}(x)-u_{p}(y)\right|^{p} d x d y\right)^{\frac{q}{p}} .
\end{aligned}
$$

Proof. First, it is not difficult to see that

$$
\begin{aligned}
& \int_{\mathbb{R}^{2}} \int_{\mathbb{R}^{2}} J(x-y)\left|u_{p}(x)-u_{p}(y)\right|^{p} d x d y \\
& \quad=2 \int_{\mathbb{R}^{2}} \int_{\mathbb{R}^{2}} J(x-y)\left|u_{p}(x)-u_{p}(y)\right|^{p-2}\left(u_{p}(x)-u_{p}(y)\right) u_{p}(x) d x d y,
\end{aligned}
$$

so that, multiplying (3.7) by $u_{p}$ and integrating over $\boldsymbol{I R}^{2}$, we obtain (3.11). Then (3.12) follows by using (3.8). The estimate (3.13) follows from the contraction principle (3.9) and the fact that $u_{p}(.+h)$ is a solution corresponding to $f(.+h)$ for any $h \in \mathbb{R}^{2}$. As to (3.15), it follows by the Hölder inequality. Indeed, setting

$$
I=\int_{\mathbb{R}^{2}} \int_{\mathbb{R}^{2}} J(x-y)\left|u_{p}(x)-u_{p}(y)\right|^{q} d x d y,
$$


we have

$$
\begin{aligned}
I & =\int_{\mathbb{R}^{2}} \int_{\mathbb{R}^{2}} J^{\frac{q}{p}}(x-y)\left|u_{p}(x)-u_{p}(y)\right|^{q} J^{\frac{p-q}{p}}(x-y) d x d y \\
& \leq\left(\int_{\boldsymbol{R}^{2}} \int_{\mathbb{R}^{2}} J(x-y)\left|u_{p}(x)-u_{p}(y)\right|^{p} d x d y\right)^{\frac{q}{p}}\left(\int_{\boldsymbol{R}^{2}} \int_{\mathbb{R}^{2}} J(x-y) d x d y\right)^{\frac{p-q}{p}} \\
& \leq\left(\int_{\mathbb{R}^{2}} \int_{\mathbb{R}^{2}} J(x-y)\left|u_{p}(x)-u_{p}(y)\right|^{p} d x d y\right)^{\frac{q}{p}}
\end{aligned}
$$

where we use the fact that $\int_{\mathbb{R}^{2}} J(x) d x=1$.

Lemma 3.4. If $f \in L^{2}\left(\boldsymbol{R}^{2}\right) \cap L^{\infty}\left(\boldsymbol{R}^{2}\right)$ is compactly supported and $u_{p} \rightarrow u$ a.e. in $\boldsymbol{R}^{2}$, then $u$ is compactly supported.

Proof. To prove the lemma, we assume without loss of generality that $\operatorname{Support}(f) \subseteq$ $B(0, R)$ for a given $R>0$, and let us prove that there exists $R^{\prime}>0$, such that

$$
\operatorname{Support}(u) \subseteq B\left(0, R^{\prime}\right) .
$$

It is not difficult to see that, for any $\lambda>0$,

$$
|f(x)| \leq d_{R}(x):=\left(\|f\|_{L^{\infty}\left(\boldsymbol{R}^{2}\right)}+\lambda(R-|x|)\right)^{+}, \quad \text { a.e. } x \in \mathbb{R}^{2} .
$$

So, using (3.7), we see that

$$
u_{p}(x)-d_{R}(x)+\frac{1}{\delta^{p-2}} \int_{\mathbb{R}^{2}} J(x-y) \Phi_{p}\left(U_{p}(x, y)\right) d y \leq 0, \quad \text { a.e. } x \in \mathbb{R}^{2},
$$

and

$$
u_{p}(x)+d_{R}(x)+\frac{1}{\delta^{p-2}} \int_{\mathbb{R}^{2}} J(x-y) \Phi_{p}\left(U_{p}(x, y)\right) d y \geq 0, \quad \text { a.e. } x \in \boldsymbol{I R}^{2},
$$

where we denote $\Phi_{p}(r)=|r|^{p-2} r$ for any $r \in \mathbb{R}$ and $U_{p}(x, y)=u_{p}(x)-u_{p}(y)$, a.e. $x, y \in \mathbb{R}^{2}$. Multiplying (3.17) by $\left(u_{p}(x)-d_{R}(x)\right)^{+}$and integrating over $\boldsymbol{R}^{2}$, we get

$$
\begin{aligned}
I & :=\iint_{\mathbb{R}^{2} \times \mathbb{R}^{2}}\left(u_{p}(x)-d_{R}(x)\right)^{+2} d x \\
& \leq-\frac{1}{\delta^{p-2}} \iint_{\boldsymbol{R}^{2} \times \mathbb{R}^{2}} J(x-y) \Phi_{p}\left(U_{p}(x, y)\right)\left(u_{p}(x)-d_{R}(x)\right)^{+} d x d y .
\end{aligned}
$$

It is not difficult to see that

$$
\begin{aligned}
& \iint_{\mathbb{R}^{2} \times \mathbb{R}^{2}} J(x-y) \Phi_{p}\left(U_{p}(x, y)\right)\left(u_{p}(x)-d_{R}(x)\right)^{+} d x d y \\
& =\frac{1}{2} \iint_{\mathbb{R}^{2} \times \mathbb{R}^{2}} J(x-y) \Phi_{p}\left(U_{p}(x, y)\right)\left(\left(u_{p}(x)-d_{R}(x)\right)^{+}-\left(u_{p}(y)-d_{R}(y)\right)^{+}\right) d x d y .
\end{aligned}
$$

Moreover, since

$$
\left(\Phi_{p}\left(U_{p}(x, y)\right)-\Phi_{p}(D(x, y))\right)\left(\left(u_{p}(x)-d_{R}(x)\right)^{+}-\left(u_{p}(y)-d_{R}(y)\right)^{+}\right) \geq 0,
$$

a.e. $(x, y) \in \boldsymbol{R}^{2} \times \boldsymbol{R}^{2}$, 
where $D(x, y)=d_{R}(x)-d_{R}(y)$ for any $x, y \in \mathbb{R}^{2}$, we deduce from (3.19) that

$$
\begin{aligned}
& \iint_{\mathbb{R}^{2} \times \mathbb{R}^{2}} J(x-y) \Phi_{p}\left(U_{p}(x, y)\right)\left(u_{p}(x)-d_{R}(x)\right)^{+} d x d y \\
& \geq \frac{1}{2} \iint_{\boldsymbol{R}^{2} \times \boldsymbol{R}^{2}} J(x-y) \Phi_{p}(D(x, y))\left(\left(u_{p}(x)-d_{R}(x)\right)^{+}-\left(u_{p}(y)-d_{R}(y)\right)^{+}\right) d x d y \\
& \geq \iint_{\mathbb{R}^{2} \times \mathbb{R}^{2}} J(x-y) \Phi_{p}(D(x, y))\left(u_{p}(x)-d_{R}(x)\right)^{+} d x d y .
\end{aligned}
$$

This implies that

$$
\begin{aligned}
I & \leq-\frac{1}{\delta^{p-2}} \iint_{\boldsymbol{R}^{2} \times \mathbb{R}^{2}} J(x-y) \Phi_{p}(D(x, y))\left(u_{p}(x)-d_{R}(x)\right)^{+} d x d y \\
& \leq \delta\|f\|_{L^{\infty}\left(\boldsymbol{R}^{2}\right)} \iint_{\mathbb{R}^{2} \times \boldsymbol{R}^{2}} J(x-y)\left|\frac{d_{R}(x)-d_{R}(y)}{\delta}\right|^{p-1} d x d y,
\end{aligned}
$$

where we use the fact that $\left\|u_{p}\right\|_{L^{\infty}\left(\boldsymbol{R}^{2}\right)} \leq\|f\|_{L^{\infty}\left(\boldsymbol{R}^{2}\right)}$. Since $\left|\nabla d_{R}(x)\right| \leq \lambda$, a.e. $x \in$ $\boldsymbol{I R}^{2}$, we have

$$
\left|d_{R}(x)-d_{R}(y)\right| \leq \lambda \varepsilon \quad \text { for any }|x-y| \leq \varepsilon,
$$

and (3.20) implies that

$$
\begin{aligned}
& \iint_{\boldsymbol{R}^{2} \times \boldsymbol{R}^{2}}\left(u_{p}(x)-d_{R}(x)\right)^{+2} d x \\
& \quad \leq\|f\|_{L^{\infty}\left(\boldsymbol{R}^{2}\right)}\left(\frac{\lambda \varepsilon}{\delta}\right)^{p-2} \iint_{\boldsymbol{R}^{2} \times \boldsymbol{R}^{2}} J(x-y)\left|d_{R}(x)-d_{R}(y)\right| d x d y .
\end{aligned}
$$

Now, we assume that $\frac{\lambda \varepsilon}{\delta}<1$ and we let $p \rightarrow \infty$, obtaining

$$
\liminf _{p \rightarrow \infty} \iint_{\mathbb{R}^{2} \times \mathbb{R}^{2}}\left(u_{p}(x)-d_{R}(x)\right)^{+2} d x=0 .
$$

Thanks to the Fatou lemma, we deduce that

$$
\iint_{\mathbb{R}^{2} \times \boldsymbol{R}^{2}}\left(u(x)-d_{R}(x)\right)^{+2} d x=0,
$$

thus

$$
u \leq d_{R}, \quad \text { a.e. in } \boldsymbol{R}^{2} .
$$

In the same way, multiplying (3.18) by $\left(-u_{p}(x)-d_{R}(x)\right)^{+}$and using the same arguments, we can prove that

$$
u(x) \geq-d_{R}(x), \quad \text { a.e. in } \boldsymbol{R}^{2} .
$$

This implies that, for any $0<\lambda<\delta / \varepsilon$,

$$
\operatorname{Support}(u) \subseteq B\left(0, R_{\lambda}^{\prime}\right), \quad \text { where } R_{\lambda}^{\prime}=R+\frac{1}{\lambda}\|f\|_{L^{\infty}\left(\boldsymbol{R}^{2}\right)} .
$$


Letting $\lambda \rightarrow \delta / \varepsilon$, we deduce (3.15) with

$$
R^{\prime}=R+\frac{\varepsilon}{\delta}\|f\|_{L^{\infty}\left(\boldsymbol{R}^{2}\right)}
$$

and the proof is complete.

LEMma 3.5. If $f \in \mathcal{C}_{0}\left(\boldsymbol{R}^{2}\right) \cap L^{2}\left(\boldsymbol{I R}^{2}\right)$, then

$$
u_{p} \rightarrow u \quad \text { in } \mathcal{C}\left(\boldsymbol{R}^{2}\right) \text { and in } L^{2}\left(\boldsymbol{R}^{2}\right),
$$

$u \in \mathcal{C}_{0}\left(\boldsymbol{R}^{2}\right)$ and $u=\boldsymbol{P}_{K_{\varepsilon}^{\delta}}(f)$.

Proof. First let us assume that $f \in \mathcal{C}_{c}\left(\boldsymbol{I R}^{2}\right)$. By using (3.13) with $q=\infty$ and the Ascoli theorem, we deduce the existence of $u \in \mathcal{C}\left(\boldsymbol{I R}^{2}\right)$ and a subsequence that we denote again by $p$ such that (3.21) is fulfilled. Moreover, thanks to (3.9) with $q=2$, we see that $u_{p} \rightarrow u$ in $L^{2}\left(\boldsymbol{R}^{2}\right)$-weak. Thus, the convergence holds in $L^{2}\left(\boldsymbol{R}^{2}\right)$. Thanks to Lemma 3.4 , we deduce that $u \in \mathcal{C}_{c}\left(\boldsymbol{I R}^{2}\right)$. Let us prove that $u \in K_{\varepsilon}^{\delta}$. Thanks to (3.12) and (3.15), we see that

$$
\begin{aligned}
I_{p} & :=\int_{\mathbb{R}^{2}} \int_{\boldsymbol{R}^{2}} J(x-y)\left|u_{p}(x)-u_{p}(y)\right|^{q} d x d y \\
& \leq\left(\int_{\boldsymbol{R}^{2}} \int_{\boldsymbol{R}^{2}} J(x-y)|| u_{p}(x)-\left.u_{p}(y)\right|^{p} d x d y\right)^{\frac{q}{p}} \\
& \leq\left(2 \delta^{\frac{q(p-2)}{p}} \int_{\mathbb{R}^{2}}|f(x)|^{2} d x\right)^{\frac{q}{p}} .
\end{aligned}
$$

Keeping $q \geq 1$ fixed, letting $p \rightarrow \infty$, and using (3.21), we deduce that

$$
\int_{\mathbb{R}^{2}} \int_{\mathbb{R}^{2}} J(x-y)\left|\frac{u(x)-u(y)}{\delta}\right|^{q} d x d y \leq 1 .
$$

Since $q \geq 1$ is arbitrary, we deduce that $|u(x)-u(y)| \leq \delta$ for $(x-y) \in \operatorname{Support}(J)=$ $B(0, \varepsilon)$. This implies that $u \in K_{\varepsilon}^{\delta}$. Now, for any $\xi \in K_{\varepsilon}^{\delta}$, let us prove that

$$
\int_{\mathbb{R}^{2}}(f(x)-u(x))(u(x)-\xi(x)) d x \leq 0 .
$$

For any $\xi \in K_{\varepsilon}^{\delta}$, it is clear that $\frac{n}{n+1} \xi \in K_{\varepsilon}^{\delta}$ for any $n \geq 1$. Taking $u-\frac{n}{n+1} \xi$ as a test function in (3.7), we see that

$$
\begin{aligned}
\int_{\mathbb{R}^{2}}\left(u_{p}(x)-\right. & f(x))\left(u_{p}(x)-\frac{n}{n+1} \xi(x)\right) d x \\
\geq & -\int_{\mathbb{R}^{2}} \int_{\mathbb{R}^{2}} J(x-y)\left|\frac{\xi(x)-\xi(y)}{\delta(n+1) / n}\right|^{p-2}\left(u_{p}(x)-\frac{n}{n+1} \xi(x)\right) d x d y .
\end{aligned}
$$

Since $\left|\frac{\xi(x)-\xi(y)}{\delta(n+1) / n}\right| \leq \frac{n}{n+1}<1$ for $|x-y| \leq \varepsilon, \operatorname{Support}(J)=B(0, \varepsilon)$, and $\left\|u_{p}\right\|_{L^{\infty}\left(\boldsymbol{R}^{2}\right)} \leq$ $\|f\|_{L^{\infty}\left(\boldsymbol{R}^{2}\right)}$

$$
\lim _{p \rightarrow \infty} \int_{\mathbb{R}^{2}} \int_{\mathbb{R}^{2}} J(x-y)\left|\frac{\xi(x)-\xi(y)}{\delta(n+1)}\right|^{p-2}\left(u_{p}(x)-\frac{n}{n+1} \xi(x)\right) d x d y=0 .
$$


So, letting $p \rightarrow \infty$ in (3.23) and using the first part of the proof, we deduce that

$$
\int_{\mathbb{R}^{2}} u(x)\left(u(x)-\frac{n}{n+1} \xi(x)\right) \geq \int_{\mathbb{R}^{2}} f(x)\left(u(x)-\frac{n}{n+1} \xi(x)\right) .
$$

Then, letting $n \rightarrow \infty$, we deduce (3.22).

Now, for any $f \in \mathcal{C}_{0}\left(\boldsymbol{R}^{2}\right)$, we consider a sequence $f_{n} \in \mathcal{C}_{c}\left(\boldsymbol{R}^{2}\right)$, such that

$$
f_{n} \rightarrow f \quad \text { in } \mathcal{C}\left(\boldsymbol{R}^{2}\right) .
$$

Let us denote by $u_{p n}$ the solution of (3.7) corresponding to $f_{n}$. Thanks to the $L^{\infty}$ contraction property of $\boldsymbol{I P}_{K_{\varepsilon}^{\delta}}$ and of the solution of (3.7), we have

$$
\begin{aligned}
\left|\boldsymbol{P}_{K_{\varepsilon}^{\delta}} f(x)-u_{p}(x)\right| \leq & \left\|\boldsymbol{P}_{K_{\varepsilon}^{\delta}} f-\boldsymbol{P}_{K_{\varepsilon}^{\delta}} f_{n}\right\|_{L^{\infty}\left(\boldsymbol{R}^{2}\right)}+\left\|\boldsymbol{P}_{K_{\varepsilon}^{\delta}} f_{n}-u_{p n}\right\|_{L^{\infty}\left(\boldsymbol{R}^{2}\right)} \\
& +\left\|u_{p n}(x)-u_{p}(x)\right\|_{L^{\infty}\left(\boldsymbol{R}^{2}\right)} \\
\leq & 2\left\|f-f_{n}\right\|_{L^{\infty}\left(\boldsymbol{R}^{2}\right)}+\left\|\boldsymbol{P}_{K_{\varepsilon}^{\delta}} f_{n}-u_{p n}\right\|_{L^{\infty}\left(\boldsymbol{R}^{2}\right)} .
\end{aligned}
$$

Thanks to the first part of the proof, it follows that

$$
\limsup _{p \rightarrow \infty} \sup _{x \in \boldsymbol{R}^{2}}\left|\boldsymbol{P}_{K_{\varepsilon}^{\delta}} f(x)-u_{p}(x)\right| \leq 2\left\|f-f_{n}\right\|_{L^{\infty}\left(\boldsymbol{R}^{2}\right)},
$$

and, by letting $n \rightarrow \infty$, we deduce again that (3.21) is fulfilled.

Lemma 3.6. If $f \in \mathcal{C}_{0}\left(\boldsymbol{R}^{2}\right) \cap L^{2}\left(\boldsymbol{R}^{2}\right)$ and $u=\boldsymbol{P}_{K_{\varepsilon}^{\delta}}(f)$, then $u$ is the solution of (3.4).

Proof. Recall that $u \in \mathcal{C}_{0}\left(\boldsymbol{R}^{2}\right)$ and

$$
u_{p} \rightarrow u \quad \text { in } \mathcal{C}\left(\boldsymbol{R}^{2}\right)
$$

where $u_{p}$ is the solution of (3.7). Let us prove that $u$ is the solution of $\left(P_{\varepsilon}^{\delta}\right)$. To that aim, we set

$$
\mu_{p}:=J(x-y)\left|\frac{u_{p}(x)-u_{p}(y)}{\delta}\right|^{p-2}
$$

and we prove that a weak ${ }^{*}$ limit of $\mu_{p}$ in $\mathcal{M}_{b}\left(\boldsymbol{R}^{2} \times \boldsymbol{R}^{2}\right)^{+}$is a kernel for the integral equation of $\left(P_{\varepsilon}^{\delta}\right)$. Thanks to (3.12) and (3.15) with $q=p-2$, we see that

$$
\iint_{\mathbb{R}^{2} \times \mathbb{R}^{2}} \mu_{p}(x, y) d x d y \leq\left(\frac{2}{\delta^{2}} \int_{\mathbb{R}^{2}}|f(x)|^{2} d x\right)^{\frac{p-2}{p}} .
$$

So, $\mu_{p}$ is bounded in $L^{1}\left(\boldsymbol{R}^{2} \times \boldsymbol{R}^{2}\right)$, and there exists $\mu \in \mathcal{M}_{b}\left(\boldsymbol{R}^{2} \times \boldsymbol{R}^{2}\right)^{+}$such that

$$
\mu_{p} \rightarrow \mu \quad \text { in } \mathcal{M}_{b}\left(\boldsymbol{R}^{2} \times \boldsymbol{R}^{2}\right)^{+} \text {-weak }{ }^{*} .
$$

In addition, since $\mu_{p}$ is symmetric, then $\mu \in \mathcal{M}_{b}^{s}\left(\boldsymbol{R}^{2} \times \boldsymbol{R}^{2}\right)^{+}$. Now, let us prove that the measure $\mu$ is supported in $[|u(x)-u(y)|=\delta$ and $|x-y| \leq \varepsilon]$. It is clear that $\mu\llcorner[|x-y| \leq \varepsilon]$. Now, let $0<\lambda<\delta$ and let $A$ be an open set such that 
$A \subseteq[\mid u(x)-u(y)<\delta-\lambda$ and $|x-y| \leq \varepsilon]$. Thanks to (3.24), there exists $p_{0}>1$, such that for any $p>p_{0}$, we have

$$
\sup _{(x, y) \in A}\left|u_{p}(x)-u_{p}(y)\right|<\delta-\lambda,
$$

so that

$$
\mu(A) \leq \lim _{p \rightarrow \infty} \iint_{A} J(x-y)\left|\frac{u_{p}(x)-u_{p}(y)}{\delta}\right|^{p-2} d x d y=0 .
$$

This implies that

$$
\mu\llcorner[|u(x)-u(y)| \geq \delta-\lambda \text { and }|x-y| \leq \varepsilon] .
$$

Since $0<\lambda<\delta$ is arbitrary, we deduce that

$$
\begin{aligned}
\operatorname{Support}(\mu) & \subseteq \cap_{0<\lambda<\delta}[|u(x)-u(y)| \geq \delta-\lambda \text { and }|x-y| \leq \varepsilon] \\
& \subseteq[|u(x)-u(y)|=\delta \text { and }|x-y| \leq \varepsilon] .
\end{aligned}
$$

Now, multiplying (3.7) by a test function $\xi \in \mathcal{C}_{c}\left(\boldsymbol{R}^{2}\right)$ and integrating over $\boldsymbol{R}^{2}$, we obtain

$$
\begin{aligned}
& \int_{\mathbb{R}^{2}} u_{p}(x) \xi(x) d x+\int_{\mathbb{R}^{2}} \int_{\mathbb{R}^{2}} J(x-y)\left|\frac{u_{p}(x)-u_{p}(y)}{\delta}\right|^{p-2}\left(u_{p}(x)-u_{p}(y)\right) \xi(x) d x d y \\
& \quad=\int_{\mathbb{R}^{2}} f(x) \xi(x) d x
\end{aligned}
$$

Thanks to (3.24), the first term of (3.26) converges to $\int_{\mathbb{R}^{2}} u(x) \xi(x) d x$ and

$$
\left(u_{p}(x)-u_{p}(y)\right) \xi(x) \rightarrow(u(x)-u(y)) \xi(x) \quad \text { uniformly for }(x, y) \in \mathbb{R}^{2} .
$$

Thus

$$
\begin{aligned}
& \lim _{p \rightarrow \infty} \int_{\mathbb{R}^{2}} \int_{\mathbb{R}^{2}} J(x-y)\left|\frac{u_{p}(x)-u_{p}(y)}{\delta}\right|^{p-2}\left(u_{p}(y)-u_{p}(x)\right) \xi(x) d x d y \\
& \quad=\int_{\mathbb{R}^{2}} \int_{\mathbb{R}^{2}}(u(y)-u(x)) \xi(x) \mu(d x, d y) .
\end{aligned}
$$

This implies that $u$ satisfies the integral equation of (3.4) for any $\xi \in \mathcal{C}_{c}\left(\boldsymbol{R}^{2}\right)$. By density, we deduce that this holds true for any $\xi \in \mathcal{C}_{0}\left(\boldsymbol{R}^{2}\right) \cap L^{2}\left(\boldsymbol{R}^{2}\right)$. Thus $u$ is a solution of (3.4).

Remark 3.7. Assuming $f \in \mathcal{C}_{0}\left(\boldsymbol{R}^{2}\right)$ guarantees that $u \in \mathcal{C}_{0}\left(\boldsymbol{I R}^{2}\right)$ and the integral formulation in (3.4) makes sense. Otherwise, $u$ is not continuous and $\mu$ is a Radon measure in general. So, one needs to see how to handle the integral formulation of the solution. This will not be treated in the present paper and may be discussed in forthcoming papers. 


\section{Proof of the main results.}

4.1. Existence and uniqueness of a solution for the PIDE. Thanks to Theorem 3.1, the proof of Theorems 2.1 and 2.2 follows by using some arguments from nonlinear semigroup theory. First, recall that

$$
\boldsymbol{P}_{K_{\varepsilon}^{\delta}}=\left(I+\lambda \partial \boldsymbol{I}_{K_{\varepsilon}^{\delta}}\right)^{-1} \quad \text { for any } \lambda>0,
$$

so that the stationary problem (3.3) is equivalent to

$$
u+\partial \mathbb{I}_{K_{\varepsilon}^{\delta}}(u) \ni f
$$

In particular, Theorem 3.1 gives the characterization of $\partial \mathbb{I}_{K_{\varepsilon}^{\delta}}$ in terms of an integral equation. This is the aim of the following corollary.

Corollary 4.1. Let $f \in \mathcal{C}_{0}\left(\boldsymbol{R}^{2}\right) \cap L^{2}\left(\boldsymbol{I R}^{2}\right)$ and $u \in \mathcal{C}_{0}\left(\boldsymbol{R}^{2}\right) \cap K_{\varepsilon}^{\delta}$. Then $f \in$ $\partial I_{K_{\varepsilon}^{\delta}}(u)$ if and only if there exists $\mu \in \mathcal{M}_{b}^{s}\left(\boldsymbol{R}^{2} \times \boldsymbol{I}^{2}\right)^{+}$such that

$$
\mu\left\llcorner\mathcal{R}_{\varepsilon}^{\delta}(u)\right.
$$

and

$$
\int_{\mathbb{R}^{2}} \int_{\mathbb{R}^{2}}(u(x)-u(y)) \xi(x) \mu(d x, d y)=\int_{\mathbb{R}^{2}} f(x) \xi(x) d x
$$

for any $\xi \in \mathcal{C}_{0}\left(\boldsymbol{R}^{2}\right) \cap L^{2}\left(\boldsymbol{R}^{2}\right)$

Proof. It is clear that $f \in \partial \mathbb{I}_{K_{\varepsilon}^{\delta}}(u)$ if and only if

$$
u=\boldsymbol{P}_{K_{\varepsilon}^{\delta}}(f+u) .
$$

Then, using Theorem 3.1, the result follows.

Corollary 4.1 suggests connecting the problem $\left(P_{\varepsilon}^{\delta}\right)$ to the evolution problem (2.6). We know, thanks to [16], that $\partial \mathbb{I}_{K_{\varepsilon}^{\delta}}$ is a maximal monotone graph in $L^{2}\left(\boldsymbol{R}^{2}\right)$, and, thanks to [11], that, for any $1 \leq q \leq \infty$, if $u_{i}$ is the solution corresponding to $f_{i} \in L^{q}\left(\boldsymbol{I R}^{2}\right)$ for $i=1,2$, then

$$
\left\|u_{1}-u_{2}\right\|_{L^{q}\left(\boldsymbol{R}^{2}\right)} \leq\left\|f_{1}-f_{2}\right\|_{L^{q}\left(\boldsymbol{R}^{2}\right)}
$$

and

$$
\left\|\left(u_{1}-u_{2}\right)^{+}\right\|_{L^{1}\left(\boldsymbol{R}^{2}\right)} \leq\left\|\left(f_{1}-f_{2}\right)^{+}\right\|_{L^{1}\left(\boldsymbol{R}^{2}\right)} .
$$

In particular this gives the concept of variational solutions for $\left(P_{\varepsilon}^{\delta}\right)$. It corresponds to the solution of the evolution equation (2.6). More precisely, for a given $f \in L_{l o c}^{2}\left(0, T ; L^{2}\left(\boldsymbol{I R}^{2}\right)\right)$ and $u_{0} \in K_{\varepsilon}^{\delta}$, we say that $u$ (resp., $\left.u_{\sigma}\right)$ is a variational solution (resp., $\sigma$-approximate variational solution) of $\left(P_{\varepsilon}^{\delta}\right)$ if $u \in W^{1,1}\left(0, T ; L^{2}\left(\boldsymbol{R}^{2}\right)\right)$, $u(0)=u_{0}$, and, for any $t \in(0, T), u(t) \in K_{\varepsilon}^{\delta}$ and

$$
\int_{\mathbb{R}^{2}}\left(f(t)-\frac{\partial u(t)}{\partial t}\right)(u(t)-z) \geq 0 \quad \text { for any } z \in K_{\varepsilon}^{\delta}
$$

(resp., $u_{\sigma}$ is given by (3.1) and $u_{i}=\boldsymbol{P}_{K_{\varepsilon}^{\delta}}\left(u_{i-1}+\sigma f^{i}\right)$ ).

By using nonlinear semigroup theory in Hilbert space for evolution problems governed by a subdifferential operator (cf. $[16,12,36]$ ), we have the following result.

Theorem 4.2. Let $u_{0} \in K_{\varepsilon}^{\delta}, T>0$, and $f \in L^{2}\left([0, T) ; L^{2}\left(\boldsymbol{I R}^{2}\right)\right.$. Then the following hold: 
1. For any $\sigma>0$ and $\sigma$-discretization of $\left(P_{\varepsilon}^{\delta}\right)$, there exists a unique $\sigma$-approximate variational solution of $\left(P_{\varepsilon}^{\delta}\right)$.

2. There exists a unique $u \in \mathcal{C}\left([0, T) ; L^{2}\left(\boldsymbol{I R}^{2}\right)\right)$ such that $u(0)=u_{0}$, and, as $\sigma \rightarrow 0$,

$$
u_{\sigma} \rightarrow u \quad \text { in } \quad \mathcal{C}\left([0, T) ; L^{2}\left(\boldsymbol{R}^{2}\right)\right) .
$$

3. If $f \in B V\left(0, T ; L^{2}\left(\boldsymbol{I R}^{2}\right)\right)$, then the function $u$ given by part 2 is the unique solution of $(2.6)$, which we call the variational solution of $\left(P_{\varepsilon}^{\delta}\right)$.

4. If, for $i=1,2, u_{i}$ is the solution (given by part 2 ) corresponding to $f_{i}$, then, for any $1 \leq q \leq \infty$,

$$
\frac{d}{d t}\left\|u_{1}-u_{2}\right\|_{L^{q}\left(\boldsymbol{R}^{2}\right)} \leq\left\|f_{1}-f_{2}\right\|_{L^{q}\left(\boldsymbol{R}^{2}\right)} \quad \text { in } \mathcal{D}^{\prime}(0, T)
$$

and

$$
\frac{d}{d t}\left\|\left(u_{1}-u_{2}\right)^{+}\right\|_{L^{1}\left(\boldsymbol{R}^{2}\right)} \leq\left\|\left(f_{1}-f_{2}\right)^{+}\right\|_{L^{1}\left(\boldsymbol{R}^{2}\right)} \quad \text { in } \mathcal{D}^{\prime}(0, T)
$$

5. In particular, if $f \geq 0$, then $u \geq 0$ a.e. in $Q$.

Proof. The first part of the theorem is a simple consequence of the fact that the variational solution $u_{i}$ of $(3.2)$ is equal to $\boldsymbol{P}_{K}\left(u_{i-1}+\varepsilon f_{i}\right)$. Since $\partial \boldsymbol{I}_{k}$ is a maximal monotone graph in $L^{2}\left(\boldsymbol{I R}^{2}\right)$, the second part of the proposition is a consequence of the classical nonlinear semigroup theory (cf. [16]; see also [12] and [36]). The third part of the proposition follows from regularity results for semigroup solutions of evolution equations governed by subdifferential operators (cf. Theorem 3.6 of [16]). Parts 4 and 5 of the theorem follow from (4.2) and (4.3), respectively.

At last, using the characterization of the operator $\partial \boldsymbol{I}_{K_{\varepsilon}^{\delta}}$ (cf. Corollary 4.1) and Theorem 4.2 , we prove the existence and uniqueness of a solution for $\left(P_{\varepsilon}^{\delta}\right)$. To this aim, we first prove the following lemma.

LEMma 4.3. Under the assumption of Theorem 2.1, let $u$ be the solution of (2.6). Then, for any $t \geq 0, u(t) \in \mathcal{C}_{0}\left(\boldsymbol{I R}^{2}\right) \cap L^{2}\left(\boldsymbol{I R}^{2}\right)$.

Proof. Coming back to the Euler implicit discretization in time of (2.6), let us consider again

$$
u_{i}=\boldsymbol{P}_{K_{\varepsilon}^{\delta}}\left(u_{i-1}+\sigma f^{i}\right) \quad \text { for } i=1, \ldots, n,
$$

where $\left(t_{i}, f_{i}\right)_{i=1, \ldots, n}$ is a $\sigma$-discretization of (2.6). We can assume without loss of generality that $f_{i} \in \mathcal{C}_{0}\left(\boldsymbol{R}^{2}\right)$ and setting

$$
\left.\left.f_{\sigma}(t)=f_{i} \quad \text { for any } t \in\right] t_{i-1}, t_{i}\right] \text { and } i=1, \ldots, n,
$$

we can assume that

$$
\lim _{h \rightarrow 0} \int_{0}^{T}\left\|f_{\sigma}(t, .+h)-f_{\sigma}(t, .)\right\|_{L^{\infty}\left(\boldsymbol{R}^{2}\right)} d t=0, \text { uniformly with respect to } \sigma .
$$

Thanks to Lemma 3.4, we deduce that $u_{i} \in \mathcal{C}_{0}\left(\boldsymbol{R}^{2}\right)$ for any $i=0,1, \ldots, n$, so that $u_{\sigma}(t) \in \mathcal{C}_{0}\left(\boldsymbol{R}^{2}\right)$ for any $t \in(0, T)$. Moreover, for any $h \in \boldsymbol{R}^{2}$, we know that

$$
\left|u_{i}(x+h)-u_{i}(x)\right| \leq\left\|u_{0}(.+h)-u_{0}(.)\right\|_{L^{\infty}\left(\boldsymbol{R}^{2}\right)}+\sum_{i=1}^{n}\left\|f_{i}(.+h)-f_{i}(.)\right\|_{L^{\infty}\left(\boldsymbol{R}^{2}\right)},
$$


so that, for any $t \in[0, T)$, we have

$$
\begin{aligned}
& \left|u_{\sigma}(t, x+h)-u_{\sigma}(t, x)\right| \leq\left\|u_{0}(.+h)-u_{0}(.)\right\|_{L^{\infty}\left(\mathbb{R}^{2}\right)} \\
& \quad+\int_{0}^{T}\left\|f_{\sigma}(t, .+h)-f_{\sigma}(t, .)\right\|_{L^{\infty}\left(\boldsymbol{R}^{2}\right)} d t \quad \text { for a.e. } x \in \mathbb{R}^{2} .
\end{aligned}
$$

Thanks to the assumption of Theorem 2.1 and (4.4), we deduce that

$$
\lim _{h \rightarrow 0} \max _{x \in \mathbb{R}^{2}}\left|u_{\sigma}(t, x+h)-u_{\sigma}(t, x)\right|=0, \text { uniformly with respect to } \sigma .
$$

Recall that $u_{\sigma} \rightarrow u$ in $\mathcal{C}\left(0, T ; L^{2}\left(\boldsymbol{R}^{2}\right)\right.$ as $\sigma \rightarrow 0$. Using (4.5) and the Ascoli theorem we deduce that $u(t) \in \mathcal{C}_{0}\left(\boldsymbol{I R}^{2}\right)$ for any $t \in[0, T)$.

LEMMA 4.4. Under the assumption of Theorem 2.1, the solution of (2.6) given by Theorem 4.2 is the unique solution of $\left(P_{\varepsilon}^{\delta}\right)$.

Proof. First, using Corollary 4.1, it is not difficult to see that a solution of $\left(P_{\varepsilon}^{\delta}\right)$ is a solution of (2.6), thus it is unique. Now, let $u$ be the variational solution of $\left(P_{\varepsilon}^{\delta}\right)$. Then $u \in W^{1, \infty}\left(0, T ; L^{2}(\Omega)\right), u(0)=u_{0}$, and $f(t)-\frac{\partial u(t)}{\partial t} \in \partial \boldsymbol{I}_{K_{\varepsilon}^{\delta}}(u(t))$, a.e. $t \in(0, T)$. Thanks to Lemma 4.3 , we know that $u(t) \in \mathcal{C}_{0}\left(\boldsymbol{R}^{2}\right)$ for any $t \in[0, T)$. Then, by using Corollary 4.1, for any fixed $t \in(0, T)$, there exists $\mu(t) \in \mathcal{M}_{b}^{s}\left(\boldsymbol{R}^{2}\right)$, such that $\mu(t)\left\llcorner\mathcal{R}_{\varepsilon}^{\delta}(u(t))\right.$ and

$$
\int_{\mathbb{R}^{2}} \int_{\mathbb{R}^{2}}(u(t, x)-u(t, y)) \xi(x) \mu(t, x, d y)=\int_{\mathbb{R}^{2}}\left(f(t, x)-\frac{\partial u(t, x)}{\partial t}\right) \xi(x) d x
$$

for any $\xi \in \mathcal{C}_{0}\left(\boldsymbol{I R}^{2}\right)$. This ends up the proof of the lemma.

Proof of Theorems 2.1 and 2.2. The proof follows directly by Theorem 4.2 and Lemma 4.4.

4.2. The connection with the stochastic model. Thanks to [23], we know that the connection between the stochastic model and the evolution surface model for a sandpile is given through the following nonlinear dynamics in $H$ :

$$
\left\{\begin{array}{l}
\partial_{t} \hat{u}+\partial \boldsymbol{I}_{\hat{K}}(\hat{u}) \ni \hat{f} \quad \text { for } t \geq 0, \\
\hat{u}(0)=0
\end{array}\right.
$$

where $H=l^{2}\left(\boldsymbol{Z}^{2}\right), \partial \boldsymbol{I}_{\hat{K}}$ denotes the subdifferential of $\boldsymbol{I}_{\hat{K}}$ in $H$, and

$$
\hat{K}:=\{\eta \in H ;|\eta(i)-\eta(j)| \leq 1 \text { if } i \sim j\} .
$$

In other words, $\hat{g} \in \partial \boldsymbol{I}_{\hat{K}}(\hat{u})$ if and only if $\hat{u} \in \hat{K}, g \in H$, and

$$
\sum_{i \in \mathbb{Z}^{2}} \hat{g}(i)(\hat{v}(i)-\hat{\xi}(i)) \geq 0 \quad \text { for any } \hat{\xi} \in H .
$$

Since $\hat{K}$ is a closed and convex subset of $H$ for a given $\hat{f} \in B V(0, T ; H)$, the evolution problem (4.7) has a unique solution (cf. [16]) in the sense that

$$
\left\{\begin{array}{l}
\hat{u} \in W^{1, \infty}(0, T ; H), \hat{u}(t) \in \hat{K} \text { a.e. } t \in[0, T), \\
\sum_{i \in \mathbb{Z}^{2}}\left(\hat{f}(t, i)-\partial_{t} \hat{u}(t, i)\right)(\hat{u}(t, i)-\hat{\xi}(i)) \geq 0, \text { a.e. } t \in[0, T), \forall \hat{\xi} \in \hat{K} .
\end{array}\right.
$$


This is a discrete analogue of (2.6), and thus the discrete analogue of $\left(P_{\varepsilon}^{\delta}\right)$. In particular, subdividing the plane into squares of side length $1 / N$, with $N \in \mathbb{I N}^{*}$, we prove the following result, which gives an explicit connection between the solution of $\left(P_{\varepsilon}^{\delta}\right)$ and the solution of (4.7).

LEMMA 4.5. Under the assumptions $\mathrm{H} 1-\mathrm{H} 3$, we assume that there exists $N \in$ $\boldsymbol{I N}^{*}$, such that (2.13) is fulfilled. Then, $u$ is a solution of $\left(P_{\varepsilon}^{\delta}\right)$, with $\varepsilon=\frac{1}{N}$ and $\delta=\frac{1}{P}$, if and only if

$$
u(t, x)=\frac{1}{P} \hat{u}(P t,[N x]) \quad \text { for any }(t, x) \in[0, \infty) \times \mathbb{R}^{2},
$$

where $\hat{u}$ is the solution of (4.7).

Proof. We know that both problems $\left(P_{\varepsilon}^{\delta}\right)$ and (4.7) have unique solutions. To prove the lemma, we show that if $\hat{u}$ is the solution of $(4.7)$, then $u(t, x):=$ $\frac{1}{P} \hat{u}(P t,[N x])$ is a solution of $\left(P_{\varepsilon}^{\delta}\right)$. It is not difficult to see that $u(t) \in K_{\varepsilon}^{\delta}$ for any $t \geq 0$. Now, for a given $\xi \in K_{\varepsilon}^{\delta}$, let us prove that

$$
\mathcal{J}:=\int_{\mathbb{R}^{2}}\left(f(t, x)-\partial_{t} u(t, x)\right)(u(t, x)-\xi(x)) d x \geq 0 .
$$

For any $i \in \mathbb{Z}^{2}$, let us denote

$$
I_{i}=\left\{z \in \boldsymbol{R}^{2} ;[N z]=i\right\} .
$$

Since for any $x \in I_{i}, u(t, x)=\delta \hat{u}(P t, i), f(t, x)=\hat{f}(P t, i)$, and $\left|I_{i}\right|=\varepsilon^{2}$, then

$$
\begin{aligned}
\mathcal{J} & =\sum_{i \in \mathbb{Z}^{2}} \int_{I_{i}}\left(f(t, x)-\partial_{t} u(t, x)\right)(u(t, x)-\xi(x)) d x \\
& =\left|I_{i}\right| \sum_{i \in \mathbb{Z}^{2}}\left(\hat{f}(P t, i)-\partial_{t} \hat{u}(P t, i)\right)\left(\frac{1}{P} \hat{u}(P t, i)-\frac{1}{\left|I_{i}\right|} \int_{I_{i}} \xi(x) d x\right) \\
& =\delta \varepsilon^{2} \sum_{i \in \mathbb{Z}^{2}}\left(\hat{f}(P t, i)-\partial_{t} \hat{u}(P t, i)\right)(\hat{u}(P t, i)-\hat{\xi}(i))
\end{aligned}
$$

where

$$
\hat{\xi}(i)=\frac{1}{\delta \varepsilon^{2}} \int_{I_{i}} \xi(x) d x
$$

It is not difficult to verify that $\hat{\xi} \in \hat{K}$, so that by using the fact that $\hat{u}$ is a solution of (4.7), we deduce that $\mathcal{J} \geq 0$.

The connection between the stochastic model and the discrete model for growing a sandpile is given by the following result.

Proposition 4.6. Assume that $\hat{f} \in B V\left(0, T ; l^{2}\left(\mathbb{Z}^{2}\right)\right), \hat{f} \geq 0$, and $f(t)$ has a bounded support in $\mathbb{Z}^{2}$ for any $t \in[0, T)$. Let $\hat{u}$ be the solution of (4.7) and let $(\eta(t), t \geq 0)$ be the stochastic process generated by $\hat{f}$. We have

$$
\boldsymbol{E}\left[\sum_{i \in \mathbb{Z}^{2}}(\eta(t, i)-\hat{u}(t, i))^{2}\right] \leq \int_{0}^{t} \sum_{j \in \mathbb{Z}^{2}} \hat{f}(s, j) d s \quad \text { for any } t \geq 0 .
$$


Recall that the proof of Proposition 4.6 (cf. [23]) is based on the inequality

$$
\sum_{j \in \mathbb{Z}^{2}} c(j, \eta, t)(\eta(t, j)-w(j)) \leq \sum_{i \in \mathbb{Z}^{2}} \hat{f}(t, i)(\eta(t, i)-w(i)) \quad \forall w \in \hat{K} .
$$

To prove (4.11), the authors of [23] defined two types of sets (type I and type II) and proved (4.11) by separating the calculation with respect to each case. Here, we use essentially the remark below and give a direct and short proof of (4.11).

Remark 4.7. For a given $\xi \in S$, if $p(i, j, \xi)>0$, then there exists at least one staircase $i_{0}=i \sim i_{1} \sim \cdots, \sim i_{m}=j$, such that $\xi\left(i_{p}\right)=\xi\left(i_{p+1}\right)+1$ for any $p=$ $0,1, \ldots, m-1$. Let us denote this staircase by $\mathcal{C}(i, j)$, i.e.,

$$
\mathcal{C}(i, j)=\left[i, i_{1}, \ldots, i_{m-1}, j\right]
$$

In addition, for $k \in \mathcal{C}(i, j)$, we denote by $\tilde{k}$ the adjacent side to $k$ satisfying $u(k)=$ $u(\tilde{k})+1$.

It is clear that for any $i, j \in \mathbb{Z}^{2}$ such that $p(i, j, \xi)>0$, and $\mathcal{C}(i, j)$ is not empty and may not be unique.

Lemma 4.8. Under the assumptions of Proposition 4.6, (4.11) is fulfilled.

Proof. Thanks to (2.8), we have

$$
\begin{aligned}
& \sum_{j \in \mathbb{Z}^{2}} c(j, \eta, t)(\eta(t, j)-w(j)) \\
& =\sum_{j, i \in \mathbb{Z}^{2}} p(i, j, \eta(t)) \hat{f}(t, i)(\eta(t, j)-w(j)) \\
& =\sum_{j, i \in \mathbb{Z}^{2}} p(i, j, \eta(t)) \hat{f}(t, i)(\eta(t, i)-w(i)) \\
& \quad+\sum_{j, i \in \mathbb{Z}^{2}} p(i, j, \eta(t)) \hat{f}(t, i)((w(i)-w(j))-(\eta(t, i)-\eta(t, j))) \\
& =I_{1}+I_{2} .
\end{aligned}
$$

Since $\sum_{j \in \mathbb{Z}^{2}} p(i, j, \eta(t))=1$ for any $(t, i) \in \mathbb{Z}^{2} \times(0, \infty)$, then

$$
I_{1}=\sum_{i \in \mathbb{Z}^{2}} \hat{f}(t, i)(\eta(t, i)-w(i)) .
$$

Let us prove that $I_{2} \leq 0$. Thanks to Remark 4.7, we have

$$
\begin{aligned}
I_{2} & =\sum_{j \neq i} p(i, j, \eta(t)) \hat{f}(t, i) \sum_{k \in \mathcal{C}(i, j) \backslash\{j\}}((w(k)-w(\tilde{k}))-(\eta(k, t)-\eta(\tilde{k}, t))) \\
& \leq \sum_{j \neq i} p(i, j, \eta(t)) \hat{f}(t, i) \sum_{k \in \mathcal{C}(i, j) \backslash\{j\}}((w(k)-w(\tilde{k}))-1)
\end{aligned}
$$

where we used the fact that $|w(k)-w(\tilde{k})| \leq 1$ (since $w \in K$ and $k \sim \tilde{k}$ ).

Remark 4.9. In the proof of (4.12), we take an arbitrary staircase $\mathcal{C}(i, j)$ associated with $p(i, j, \eta(t))>0$. The proof is independent of the choice of $\operatorname{such} \mathcal{C}(i, j)$. 
Now, the rest of the proof of Proposition 4.6 follows the same ideas of [23]. For completeness, let us give the arguments.

Proof of Proposition 4.6.

Claim 1. For any $w \in S$ and $t \geq 0$, we have

$$
I:=\frac{1}{2} \mathcal{L}_{t}\left(\sum_{i \in \mathbb{Z}^{2}}(\eta(t, i)-w(i))^{2}\right) \leq \sum_{j \in \mathbb{Z}^{2}} \hat{f}(t, j)(\eta(t, j)-w(j))+\frac{1}{2} \sum_{j \in \mathbb{Z}^{2}} f(t, j) .
$$

By definition of $\mathcal{L}_{t}$, we have

$$
I=\frac{1}{2} \sum_{j \in \mathbb{Z}^{2}} c(j, \eta, t)\left(\sum_{i \in \mathbb{Z}^{2}}\left(T_{j}(\eta(t))(i)-w(i)\right)^{2}-\sum_{i \in \mathbb{Z}^{2}}(\eta(t, i)-w(i))^{2}\right) .
$$

So,

$$
\begin{aligned}
I & =\frac{1}{2} \sum_{j \in \mathbb{Z}^{2}} c(j, \eta, t) \sum_{i \in \mathbb{Z}}\left(T_{j}(\eta)(i)-\eta(i)\right)\left(T_{j}(\eta)(i)+\eta(i)-2 w(i)\right) \\
& =\frac{1}{2} \sum_{j \in \mathbb{Z}^{2}} c(j, \eta, t)(2 \eta(j)+1-2 w(j)) \\
& =\sum_{j \in \mathbb{Z}^{2}} c(j, \eta, t)(\eta(j)-w(j))+\frac{1}{2} \sum_{j \in \mathbb{Z}^{2}} c(j, \eta, t) .
\end{aligned}
$$

Thanks to (2.9), we deduce that

$$
I=\sum_{j \in \mathbb{Z}^{2}} c(j, \eta, t)(\eta(j)-w(j))+\frac{1}{2} \sum_{j \in \mathbb{Z}^{2}} f(t, j) .
$$

Thus, the claim follows from Lemma 4.8.

Claim 2. For any $w \in W^{1, \infty}(0, T ; H)$ such that, for any $t \in[0, T), w(t) \in \hat{K}$, we have

$$
\begin{aligned}
\frac{1}{2} \sum_{i \in \mathbb{Z}^{2}}(\eta(t, i)-w(t, i))^{2} \leq & \int_{0}^{t}\left[\sum_{i \in \mathbb{Z}^{2}} w_{s}(i, s)(w(i, s)-\eta(i, s))\right. \\
& \left.+\sum_{j \in \mathbb{Z}^{2}} f(s, j)(\eta(s, j)-w(s, j))+\frac{1}{2} \sum_{j \in \mathbb{Z}^{2}} f(s, j)\right]+\mathcal{M}(t),
\end{aligned}
$$

where $\mathcal{L}_{s}$ is given by $(2.10)$ and $(\mathcal{M}(t))_{t \geq 0}$ is a martingale satisfying

$$
\boldsymbol{E}(\mathcal{M}(t))=0 \quad \text { for any } t \geq 0 .
$$

As in [23], we use the following stochastic integral equation: for any $F: S \times(0, \infty) \rightarrow$ $\boldsymbol{I R}$ Lipschitz continuous in $t$ and $F(\eta(., 0), 0)=0$, we have

$$
F(\eta(., t), t)=\int_{0}^{t}\left(\frac{\partial F}{\partial s}+\mathcal{L}_{s} F\right)(\eta(., s))+\mathcal{M}(t),
$$

where $(\mathcal{M}(t))_{t \geq 0}$ is a martingale satisfying $\boldsymbol{E}(\mathcal{M}(t))=0$ for any $t \geq 0$. Let $F$ be given by

$$
F(\xi, t)=\frac{1}{2} \sum_{i \in \mathbb{Z}^{2}}(\xi(i)-w(t, i))^{2} \quad \text { for any }(\xi, t) \in S \times(0, T) .
$$


Then

$$
\frac{\partial F}{\partial s}(\xi, s)=-\sum_{i \in \mathbb{Z}^{2}} w_{s}(i, s)(\xi(i)-w(i, s)) \quad \text { for any }(\xi, t) \in S \times(0, T),
$$

and (4.13) implies that, for any $t \geq 0$,

$$
\begin{aligned}
\frac{1}{2} & \sum_{i \in \mathbb{Z}^{2}}(\eta(t, i)-w(t, i))^{2} \\
& =\int_{0}^{t}\left(\sum_{i \in \mathbb{Z}^{2}} w_{s}(i, s)(w(i, s)-\eta(i, s))+\mathcal{L}_{s}(F(\eta(., s), s))\right) d s+\mathcal{M}(t) .
\end{aligned}
$$

Then, by using the first step, the second part of the lemma follows.

Claim 3. We have (4.10). Since $\hat{u}$ is a solution of (4.7) and $\eta(t) \in \hat{K}$ for any $t \geq 0$, we have

$$
\sum_{i \in \mathbb{Z}^{2}} \hat{u}_{s}(i, s)(\hat{u}(i, s)-\eta(i, s))+\sum_{j \in \mathbb{Z}^{2}} \hat{f}(s, j)(\eta(s, j)-\hat{u}(s, j)) \leq 0 \quad \text { for any } t \geq 0 .
$$

Then, using Claim 2 with $w=\hat{u}$, where $\hat{u}$ is the solution of (4.7), we deduce (4.10). The proof of the proposition is complete.

Proof of Theorem 2.4. Let us consider $u_{P, N}$ the solution of the PIDE associated with $f_{N}$ given by

$$
f_{N}(t, x)=f\left(t, \frac{[N x]}{N}\right) \quad \text { for any }(t, x) \in(0, \infty) \times \mathbb{R}^{2} .
$$

Setting

$$
I:=\left(\boldsymbol{E}\left[\int_{\mathbb{R}^{2}}\left|u(t, x)-\eta_{P, N}(t, x)\right|^{2}\right]\right)^{\frac{1}{2}}
$$

and using the Jensen inequality, it is not difficult to see that

$$
I \leq\left(\boldsymbol{E}\left[\int_{\mathbb{R}^{2}}\left|u_{P, N}(t, x)-\eta_{P, N}(t, x)\right|^{2}\right]\right)^{\frac{1}{2}}+\left(\int_{\mathbb{R}^{2}}\left|u_{P, N}(t, x)-u(t, x)\right|^{2}\right)^{\frac{1}{2}}
$$

For the last term of (4.14), we use the second part of Theorem 2.1 to obtain

$$
\int_{\mathbb{R}^{2}}\left|u(t, x)-u_{P, N}(t, x)\right|^{2} d x \leq \int_{0}^{t} \int_{\mathbb{R}^{2}}\left|f(t, x)-f_{N}(s, x)\right|^{2} d x d s .
$$

As for the first term on the right-hand side of (4.14), since $f_{N}$ satisfies (2.13), by using Lemma 4.5 and the fact that $\left|I_{i}\right|=\varepsilon^{2}$, we get

$$
\begin{aligned}
\boldsymbol{E}\left[\int_{\mathbb{R}^{2}}\left(\eta_{P, N}(t, x)-u_{P, N}(t, x)\right)^{2} d x\right] & =\sum_{i \in \mathbb{Z}^{2}} \boldsymbol{E}\left[\int_{I_{i}}\left(\eta_{P, N}(t, x)-u_{P, N}(t, x)\right)^{2} d x\right] \\
& =\delta^{2} \varepsilon^{2} \boldsymbol{I}\left[\sum_{i \in \mathbb{Z}^{2}}(\eta(P t, i)-\hat{u}(P t, i))^{2}\right] .
\end{aligned}
$$


So, by using (4.10), we obtain

$$
\begin{aligned}
\mathbb{E}\left[\int_{\mathbb{R}^{2}}\left(\eta_{P, N}(t, x)-u_{P, N}(t, x)\right)^{2} d x\right] & \leq \delta^{2} \varepsilon^{2} \int_{0}^{P t} \sum_{j \in \mathbb{Z}^{2}} \hat{f}(s, j) d s \\
& \leq \delta^{2} \varepsilon^{2} \int_{0}^{P t} \sum_{j \in \mathbb{Z}^{2}} f\left(\frac{s}{P}, j\right) d s \\
& \leq \delta^{2} \int_{0}^{P t} \int_{\mathbb{R}^{2}} f_{N}\left(\frac{s}{P}, x\right) d x d s \\
& \leq \delta \int_{0}^{t} \int_{\mathbb{R}^{2}} f_{N}(s, x) d x d s .
\end{aligned}
$$

Thus, (4.14) implies

$$
I \leq \delta^{\frac{1}{2}}\left(\int_{0}^{t} \int_{\boldsymbol{R}^{2}} f_{N}(s, x) d x d s\right)^{\frac{1}{2}}+\left(\int_{0}^{t} \int_{\mathbb{R}^{2}}\left|f(t, x)-f_{N}(s, x)\right|^{2} d x d s\right)^{\frac{1}{2}}
$$

and the proof is complete.

\section{REFERENCES}

[1] G. Alberti and G. Bellettini, A nonlocal anisotropic model for phase transition. Part I: The optimal profile problem, Math. Ann., 310 (1998), pp. 527-560.

[2] G. Alberti and G. Bellettini, A nonlocal anisoptropic model for phase transition: Asymptotic behaviour of rescaled, European Jl. Appl. Math., 9 (1998), pp. 261-284.

[3] F. Andreu, J. M. Mazon, J. D. Rossi, and J. Toledo, The limit as $p \rightarrow \infty$ in a nonlocal p-Laplacian evolution equation. A nonlocal approximation of a model for sandpiles, Calc. Var. Partial Differential Equations, 35 (2009), pp. 279-316.

[4] F. Andreu, J. M. Mazón, J. D. Rossi, And J. Toledo, A nonlocal p-Laplacian evolution equation with nonhomogeneous Dirichlet boundary conditions, SIAM J. Math. Anal., 40 (2009), pp. 1815-1851.

[5] D. Applebaum, Lévy processes and stochastic calculus, Cambridge Stud. Adv. Math. 93, Cambridge University Press, Cambridge, UK, 2004.

[6] I. S. Aranson and L. S. Tsimring, Patterns and collective behavior in granular media: Theoretical concepts, Rev. Modern Phys., 78 (2006), pp. 641-692.

[7] G. Aronson, L. C. Evans, And Y. Wu, Fast/slow diffusion and growing sandpiles, J. Differential Equations, 131 (1996), pp. 304-335.

[8] J. W. Barrett and L. Prigozhin, Dual formulation in critical state problems, Interfaces Free Bound., 8 (2006), pp. 349-370.

[9] D. Benedetto, E. Caglioti, and M. Pulvirenti, A kinetic equation for granular media, RAIRO Model. Math. Anal. Numer., 31 (1997), pp. 615-641.

[10] D. Benedetto, E. Caglioti, and M. Pulvirenti, Collective behavior of one-demensional granular media, in Modeling in Applied Sciences: A Kinetic Theory Approachs, N. Bellomo and M. Pulvirenti, eds., Birkhäuser Boston, Boston, MA, 2000.

[11] P. Bénilan and M. G. Crandall, Completely accretive operators, in Semigroup Theory and Evolution Equations (Delft, 1989), Lecture Notes in Pure and Appl. Math. 135, Marcel Dekker, NY, 1991, pp. 41-75.

[12] P. Bénilan, M. G. Crandall, And A. Pazy, Evolution Equations Governed by Accretive Operators, preprint.

[13] I. H. Biswas, E. R. Jakobsen, and K. H. Karlsen, Error estimates for finite differencequadrature schemes for fully nonlinear degenerate parabolic integro-PDEs, J. Hyperbolic Differ. Equ., 5 (2008), pp. 187-219.

[14] M. Bodnar and J. J. L. Velazquez, An integro-differential equation arising as a limit of individual cell-based models, J. Differential Equations, 222 (2006), pp. 341-380. 
[15] J. P. Bouchaud, M. E. Cates, J. Ravi Prakash, and S. F. Edwards, A model for the dynamics of sandpile surfaces, J. Phys. I France, 4 (1994), pp. 1383-1410.

[16] H. BRÉZIS, Opérateurs maximaux monotones et semigroupes de contractions dans les espaces de Hilbert, North-Holland Math. Stud. 5, North-Holland, Amsterdam, London, New York, 1973.

[17] C. Carrillo and P. Fife, Spatial effects in discrete generation population models, J. Math. Biol., 50 (2005), pp. 161-188.

[18] G. CRASTA AND S. FinZI Vita, An existence result for the sandpile problem on flat tables with walls, Network and Heterogeneous Media, 3 (2008), pp. 815-830.

[19] D. DhaR, Self-organized critical state of sandpile automation models, Phys. Rev. Lett., 64 (1990), pp. 1613-1616.

[20] S. Dumont AND N. IGBIDA, Back on a dual formulation for the growing sandpile problem, European J. Appl. Math., 20 (2008), pp. 169-185.

[21] S. Dumont and N. IGBida, On the collapsing sandpile problem, submitted.

[22] L. C. Evans, M. Feldman, And R. F. Gariepy, Fast/slow diffusion and collapsing sandpiles, J. Differential Equations, 137 (1997), pp. 166-209.

[23] L. C. Evans and F. Rezakhanlou, A stochastic model for sandpiles and its continuum limit, Comm. Math. Phys., 197 (1998), pp. 325-345.

[24] M. FAlCONe and S. Finzi Vita, A finite-difference approximation of a two-layer system for growing sandpiles, SIAM J. Sci. Comput., 28 (2006), pp. 1120-1132.

[25] P. Fife, Some nonclassical trends in parabolic and parabolic-like evolutions, in Trends in Nonlinear Analysis, Springer, Berlin, 2003, pp. 153-191.

[26] N. Fournier and P. Laurencot, Well-posedness of Smoluchowski's coagulation equation for a class of homogeneous kernels, J. Funct. Anal., 233 (2006), pp. 351-379.

[27] G. Gilboa And S. Osher, Nonlocal Linear Image Regularization and Supervised Segmentation, UCLA CAM Report, 2006, pp. 6-47.

[28] K. P. Hadeler And C. Kuttler, Dynamical models for granular matter, Granular Matter, 2 (1999), pp. 9-18.

[29] N. IGBidA, A generalized collapsing sandpile model, Arch. Math. (Basel), 94 (2010), pp. 193200.

[30] N. IGBIDA, Evans-Rezakhanlou stochastic model revisited, in Recent Developments in Nonlinear Analysis, Proceedings of the Conference in Mathematics and Mathematical Physics, Morocco, 2008.

[31] N. IGBIDA, Equivalent formulations for Monge-Kantorovich equation, Nonlinear Anal., 71 (2009), pp. 3805-3813.

[32] S. Kindermann, S. Osher, And P. W. Jones, Deblurring and denoising of images by nonlocal functionals, Multiscale Model. Simul., 4 (2005), pp. 1091-1115.

[33] A. Mogilner and L. Edelstein-Keshet, A non-local model for a swarm, J. Math. Biol., 38 (1999), pp. 534-570.

[34] L. Prigozhin, Variational model of sandpile growth, European J. Appl. Math., 7 (1996), pp. 225-236.

[35] H. Puhl, On the modelling of real sand piles, Phys. A, 182 (1992), pp. 295-308.

[36] R. E. Showalter, Monotone Operators in Banach Space and Nonlinear Partial Differential Equations, Math. Surveys Monogr., AMS, Providence, RI, 1997. 